Sādhanā Vol. 38, Part 6, December 2013, pp. 1173-1217. (C) Indian Academy of Sciences

\title{
Entrainment phenomenon in gas-liquid two-phase flow: A review
}

\author{
R K BAGUL $^{1,2}$, D S PILKHWAL ${ }^{1}$, P K VIJAYAN $^{1,2}$ and \\ J B JOSHI ${ }^{2,3, *}$
}
${ }^{1}$ Reactor Engineering Division, Bhabha Atomic Research Centre, Trombay, Mumbai 400 085, India
${ }^{2}$ Homi Bhabha National Institute, Anushaktinagar, Mumbai 400 094, India
${ }^{3}$ Institute of Chemical Technology, Matunga, Mumbai 400 019, India
e-mail: vijayanp@barc.gov.in; jbjoshi@gmail.com

MS received 23 April 2012; revised 14 March 2013; accepted 20 March 2013

\begin{abstract}
The gas-liquid separation equipments are aimed to be designed for maximum efficiency of phase separation. In order to maximize their capacity the flow rates are required to be optimized for the capital cost of equipment. This leads to the situation where the gas phase leaves the separation interface with high velocities and carry liquid phase along with it in the form of droplets reducing the equipment efficiency. This is known as entrainment or carryover. Depending on the nature of the separation interface i.e., turbulence intensity, bubble dynamics, the size and velocity distribution of liquid fragments, droplets at the separation interface varies. This is the main source of empiricism involved in the analysis of such equipments. The mechanics of motion of the dispersed liquid phase in bulk of gas is relatively well studied. In the present paper the various experimental, analytical and numerical investigations carried out to address the issues of entrainment/carryover are carefully analyzed. Further, a critical review has been presented for bringing out a coherent theme and a current status of the subject under reference.
\end{abstract}

Keywords. Carryover; entrainment; gas-liquid phase separation.

\section{Introduction}

Entrainment/carryover is a phenomenon associated with systems in which the liquid and gases or vapours are in relative motion. It may be defined as pneumatic conveyance of drops of liquid into gaseous phase. It has been extensively observed in number of process equipments where phase separation takes place due to gravity such as industrial boilers, evaporators, distillation columns and also in Nuclear reactors. In a bubbling pool, when the vapour bubble leaves the free surface, the bursting of bubble or/and jetting of vapour leads to formation of liquid droplets

*For correspondence 
which may get carried along with the vapour/gas flow. The liquid droplets carried along by gas (carryover) may cause mechanical damage to process equipments due to dissolved salts carried by liquid droplets along the steam, reduction in separation efficiency in evaporation, distillation or absorption columns, severe damage in turbines and transport of volatile radionuclides in case of steam generators for nuclear reactors. Carryover depends on various factors such as size and velocity of droplets, bulk velocity and flow pattern of the vapour phase, nature of forces on the droplet moving relative to vapour phase, fluid properties, viz., surface tension, viscosity, density, bubble dynamics at and below the separation interface, etc. Carryover effects can be reduced by using extra equipments like steam washers, steam dryers and mist separators. It is however important to understand the effect of factors affecting phenomenon of carryover to arrive at equipment design with minimum carryover in first place at separation interface. There is always an emphasis on the simplification of process equipments by eliminating extra units of steam separators, dryers, etc. Particularly, the advanced steam generator designs are being sought to have phase separation due to gravity, and it has to be ensured that steam leaving the generator has liquid entrainment fraction lower than the acceptable limits.

Estimation of carryover in the design of new equipment is still based on the empirical correlations developed upon the experimental data. The chronological development of the subject has occurred in both: experimental and analytical approaches culminating into the empirical correlations. Computational fluid dynamics is relatively new and has not yet been extensively applied to simulate the carryover phenomenon.

Davis (1940) first addressed the issues related to steam contamination due to the soluble salts carried by liquid entrainment in case of industrial high pressure boilers. The methodology adopted to predict the limiting vapour flow rate to minimise the entrainment was based on several assumptions upon bubble dynamics below and at the separation interface. Simplified assumptions were made regarding the droplet formation mechanism and the quantification of droplet velocity and size distribution and mechanics of droplet motion. The similar methodology was followed by Zenz \& Weil (1958) for predicting particle entrainment from fluidised beds. Cheng \& Teller (1961) analysed carryover in sieve trays. All the analytical work was stressed on the improvement in the understanding of the droplet size and velocity distribution functions with a strong experimental database. Newitt et al (1954), Garner et al (1954) studied the mechanism of droplet formation and its quantification to analyse the carryover. Spiel (1994), Aiba \& Yamada (1959), Rozen et al (1970) performed experiments of the droplet size and velocity distribution. Most of the experiments were performed in either air-water system where optical measurement was possible or in steam-water environment at low pressures. Sterman (1958) and Kolkolostev (1952) measured carryover in steam-water system at high pressure and temperature. Experiments by Mochizuki \& Hirao (1993) were relevant to the actual steam separator geometry and to estimate its performance. Cosandy et al (2001) also performed experiments being specific to the geometry under consideration. Kim \& No (2005) proposed empirical correlations for carryover in two-phase systems based on the experiments. Iyer et al (2010) performed experiments in air-water system with geometry relevant to steam drum and proposed a modified correlation for carryover.

There have been various attempts to produce a unique correlation for carryover based on the dimensional analysis and experimental database, for example, Sterman (1958). Ishii \& Kataoka (1984) developed a detailed mechanistic model, taking into account various influencing phenomena and parameters and produced a correlation for carryover which satisfied most of the experimental database available till the date. The approach and correlations provided by Ishii \& Kataoka (1984) have been used to predict the entrainment fractions by Nayak et al (2000) and Koch et al (2000) in their respective geometries under consideration. 
From the foregoing discussion, it is clear that, a good number of papers have been published to address the issue of entrainment. It was thought desirable to classify the published papers into four categories: (a) mathematical description of the phenomenon using analytical models, (b) experimental studies, (c) development of empirical correlations and (d) recent studies on numerical models. All these classes papers have been analysed and critically reviewed in the following sections 2, 3, 4 and 5 .

\section{Analytical development}

Davis (1940) addressed the issues related to the production of steam at high pressures in industrial boilers and the problems of mechanical failure of components due to salts deposited by the entrainment carried by the steam. He studied the bubble generation at the heater tubes, their movement in the pool towards the separation surface and the behaviour of steam bubbles at the free level. When a bubble arrives at the free level, it forms a nearly complete hemispherical dome shaped lamina of liquid and remains floated with stability for certain time duration before rupture.

Figure 1 shows the various structures of the surface bubbles as visualized by Davis (1940).

The hemispherical shape is due to the formation of a thin water film which is a result of balance between the excess pressure inside the bubble and the surface tension.

Size of bubble dome is given by:

$$
P_{1}-P_{0}=\frac{8 \sigma}{D_{B}}
$$

Davis (1940) has proposed the following stepwise mechanism for the droplet generation:

(a) when the bubble dome ruptures the rim of the bubble sinks

(b) which leads to rise of the depressed portion at the bubble centre due to momentum of inflowing liquid

(c) it throws a spout upwards

(d) the tip of which necks of under the action of surface tension into separate drops.

The above mechanism is explained in figure 2. The shape of the stabilized bubble at the free surface it can be observed that small bubble (diameter less than $2 \mathrm{~mm}$ ) depress the liquid sufficiently in proportion to their size. In the case of small bubbles as the bubble dome ruptures, the liquid at the rim have sufficient momentum to cause the rise of centre of depression. Large bubbles do not depress the liquid sufficiently to produce the droplet by the above described mechanism. By approximations it can be shown that the height to which the droplet would be

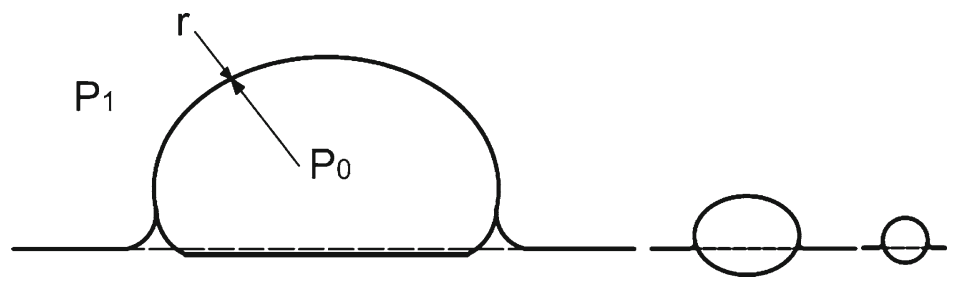

Figure 1. Structure of floating bubbles at separation interface (taken from Davis 1940). 


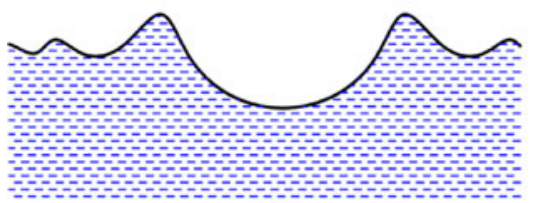

(a)

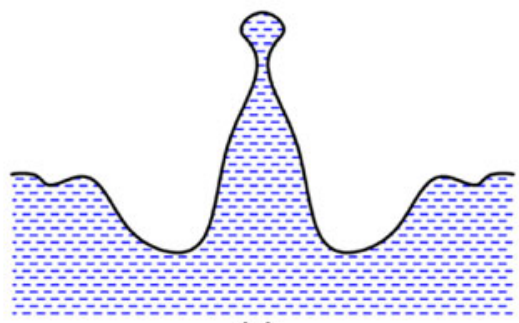

(c)

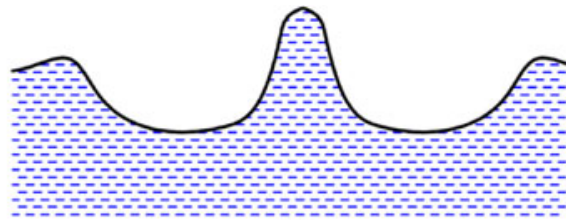

(b)

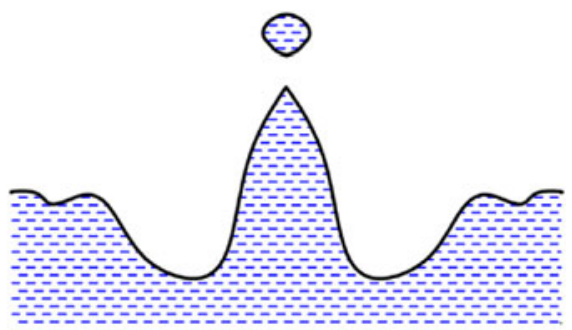

(d)

Figure 2. Mechanism for jet droplet formation: (a) Bubble rim sinks following the rupture of bubble dome, (b) the central depressed portion rises up due to momentum of liquid, (c) a spout is thrown upwards and (d) the tip of the sprout necks under action of surface tension and a droplet is formed (taken from Davis 1940).

ejected is inversely proportional to the square of bubble radius and is given by the following equation:

$$
h_{m} \propto\left(\frac{4 \sigma t_{b}}{\rho_{f} D_{B}}\right)^{2} .
$$

Davis (1940) underlined this as an important fact related to subject of carryover. Davis (1940) pointed that the height up to which the droplet can rise depends on the droplet velocity after formation and resistance of the vapour while rising. He developed equation based on the force balance for the droplet to predict the maximum height gained by a droplet in vapour space.

Considering force balance on the droplet, during upward flight of droplet we can write

$$
\frac{W}{g} \frac{d V_{r}}{d t}=-K V_{r}^{2}-W
$$

And during downward motion of droplet

$$
\frac{W}{g} \frac{d V_{r}}{d t}=K V_{r}^{2}-W .
$$

At height $y$ from free surface at time $t$, it can be written as

$$
V_{r}=\frac{d y}{d t},
$$

hence

$$
\frac{d V_{r}}{d t}=V_{r} \frac{d V_{r}}{d y} .
$$


Using above equation and integrating, it can be found that

$$
h_{m}=\frac{q^{2}}{2 g}\left[\ln \left\{1+\frac{\left(V_{i}-V_{g}\right)^{2}}{q^{2}}\right\}-\ln \left\{1-\frac{V_{g}^{2}}{q^{2}}\right\}\right]+\frac{V_{g} q}{2 g}\left[\tan ^{-1}\left(\frac{V_{i}-V_{g}}{q}\right)+\ln \left(\frac{q+V_{g}}{q-V_{g}}\right)\right] \text {, }
$$

where $q$ is terminal velocity of droplet and is given as

$$
q^{2}=\frac{W}{K} .
$$

It can be seen from Equation (6) that the droplet having terminal velocity equal or very close to vapour velocity gets carried all the way in the vapour flow, which happens to be the minimum amount of carryover that will always be present in the flow. The above expression requires the knowledge of velocity of projection and diameter of droplet and the terminal velocity of droplet i.e., drag coefficient during the various stages of motion. Davis (1940) pointed out the importance of the experimental evidence for validation. Davis (1940) made several simplifications and based on the observations of earlier experiments deduced that the average velocity of projection of droplets and was taken as $1.4 \mathrm{~m} / \mathrm{s}$. Based on the knowledge of bubble size at the heater tubes and their growth while reaching the free surface he could deduce the bubble diameter and the corresponding droplet dimension. Using Equation (6) he predicted the quantity of carryover for different steam flow rates. Davis (1940) also calculated the permissible velocities or steam rates at various pressures (see figure 3).

Davis (1940) estimated that the calculations had a factor of safety of 4 when compared with the actual entrainment at the exit of boiler. It may be noted that Davis (1940) attempted to obtain the entrainment rates based on the force balance for the droplets. However, the size and velocity distribution of droplets and their generation rates at the free surface was not completely understood at that point of time. Davis (1940) has further pointed out that the entrainment depends on

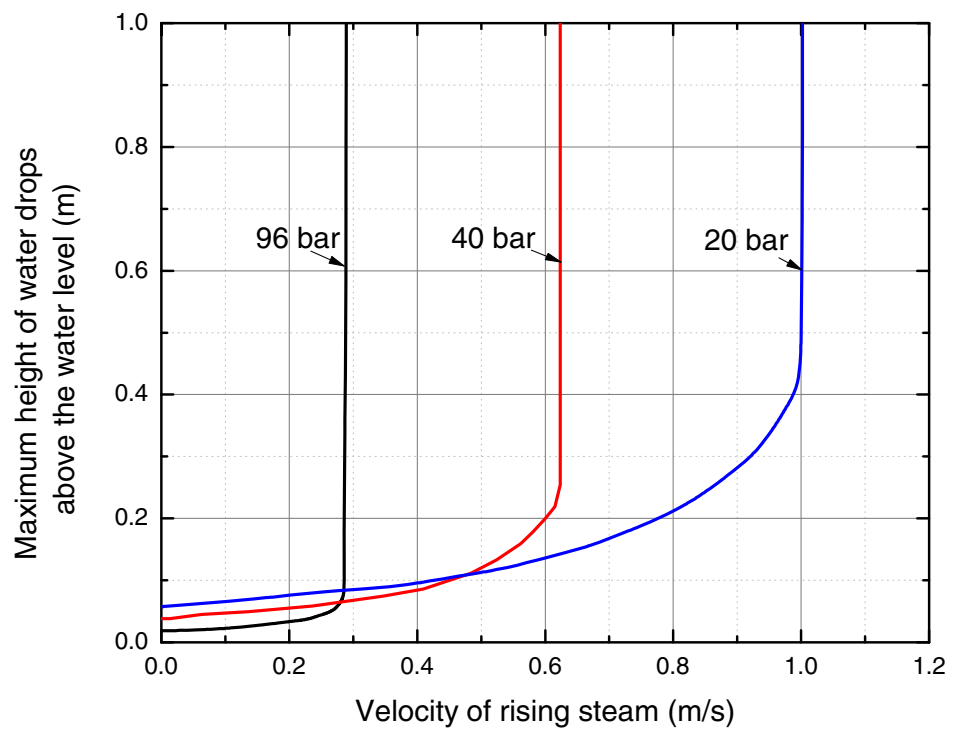

Figure 3. Height of projection of water drop at different steam velocities and pressures (taken from Davis 1940). 
the bubble dynamics in the pool below. He has also brought out the importance of the design of internals in the phenomena of carryover. Thus it may be pointed out that Davis (1940) has made pioneering contribution to the subject of entrainment.

Cheng \& Teller (1961) followed the approach developed by Davis (1940) to analyze behaviour of entrained particles in the zone between trays in a sieve tray column for the air-water system. The droplet size distribution was experimentally obtained and was found to have log-normal distribution. In the absence of velocity data, the velocities of drops were assumed/deduced from the experiments of Aiba \& Yamada (1959). The carryover estimation was found to agree within $28 \%$ when compared with the experimental measurements.

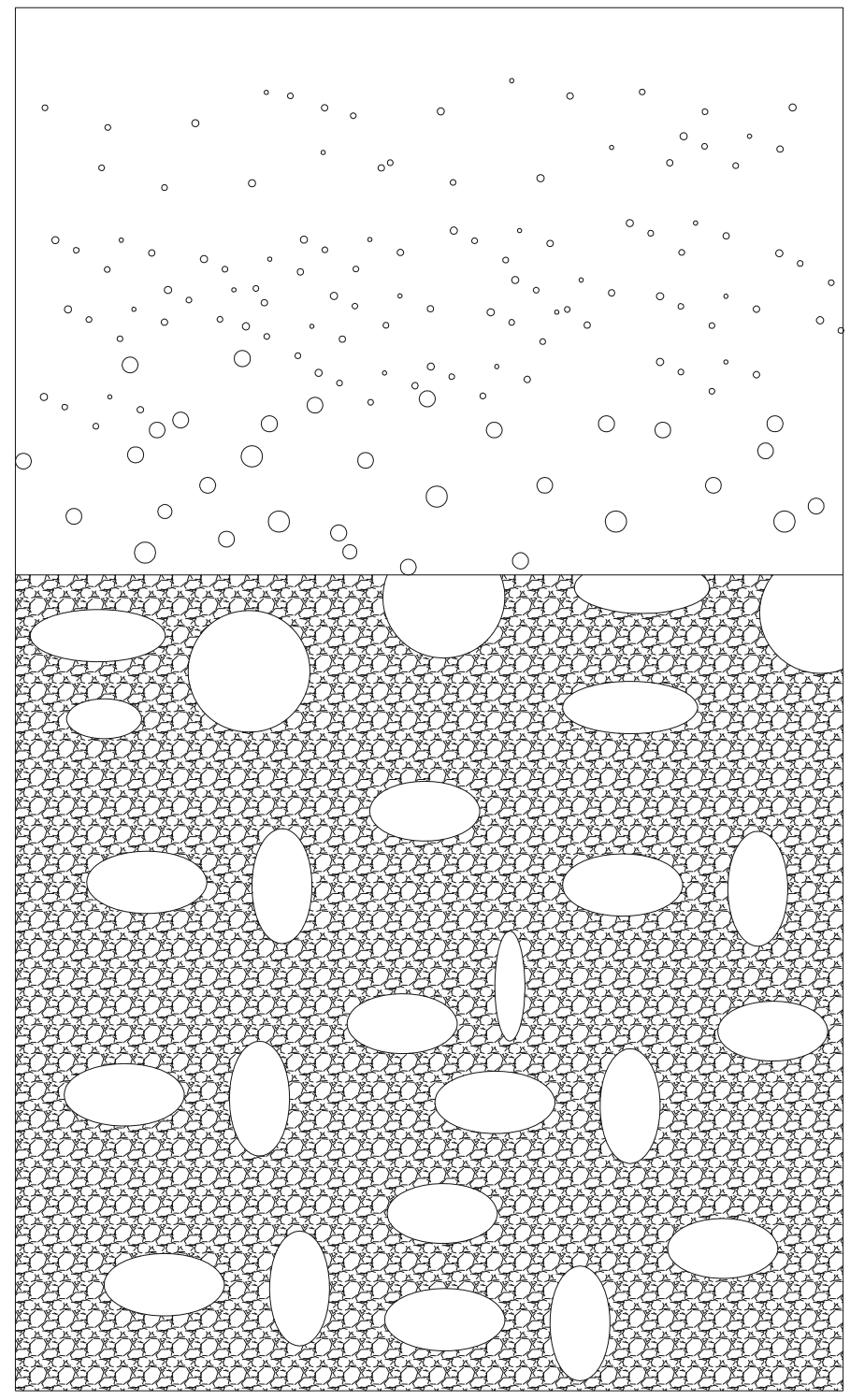

Figure 4. Schematic representation of entrainment and dispersion in gas-solid fluidization. 


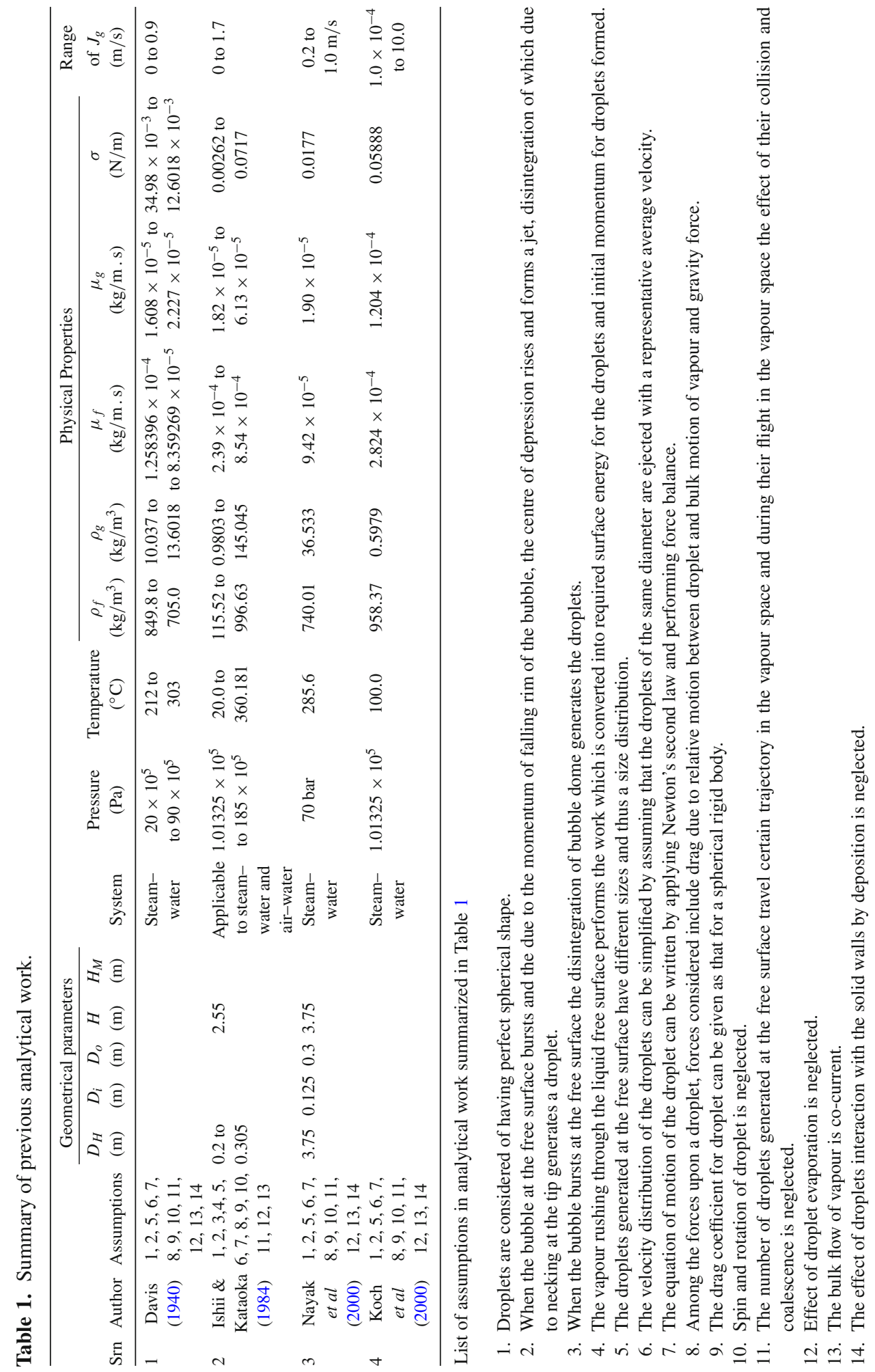


Zenz \& Weil (1958) addressed the problem of particle entrainment in fluidized bed. Figure 4 shows schematic representation of the phenomenon. An equation of motion for the solid particles was formulated for instantaneous velocity of particle, terminal velocity of particle and maximum height attained by a particle using similar approach developed by Davis (1940). However, to obtain the complete entrainment gradient pattern, the entrainment rate for various size particles must be known at the free surface. The entrainment rate at free surface was empirically modelled by authors.

Nayak et al (2000) analytically studied the carryover of water droplets by the steam in a $3.5 \mathrm{~m}$ diameter, horizontal steam drum. The steam-water separation by gravity was modelled. The droplet size and velocity distribution at the separation interface was assumed and trajectory of the droplets was computed. The droplet size distribution suggested by Kocamustafagullari et al (1993) was used in the calculation. The maximum droplet size and ejection velocity distribution was estimated based on the mechanistic model given by Ishii \& Kataoka (1984). Droplet dynamics considered the equation of motion of drop as a perfect sphere under the action of drag and buoyancy forces in a constant velocity field. Parametric study was carried out to predict the critical droplet diameter and carryover for the various steam flow rates. Nayak et al (2000) concluded that carryover is less than $0.1 \%$ when steam velocity at separation interface is less than $0.7 \mathrm{~m} / \mathrm{s}$. The analysis however did not consider the effect of change of flow cross-section and acceleration of steam flow towards the exit. The various analytical studies conducted on carryover and the various assumptions made are summarized in table 1. From above analytical work carried by various researchers, it can be noted that the subject of droplet size distribution, velocity distribution and number of parameters affecting it including the geometries is complex. However to obtain initial estimations, simplified approach as given by Davis (1940) can be followed with knowing the fact that empirical models involved can lead to conservative designs.

\section{Experimental work}

Newitt et al (1954) carried out experimental studies on bubble bursting at the air-water free surface. They studied the bubble behaviour with photography techniques. They measured the droplet size distribution using microscope slides coated with magnesium oxide and measuring the impression made by the droplets after impact on the coating (measurement accuracy $20 \mu$ ). The entrainment was obtained at different heights above the pool surface. The authors proposed the following stepwise mechanisms:

(a) Disintegration of liquid film of stable bubble at the free surface (film drops).

(b) Fragmentation of jet ejected due to rushing liquid as bubble rim sinks and centre of depression rises (jet drops).

Figure 5 shows the experimental observations by Newitt et al (1954) which shows the existence of film droplets of small diameters with high frequency of generation and large sized jet droplets with lower frequency of generation.

Newitt et al (1954) further compared the jet droplet size observed experimentally with the theoretical analysis. The jet formed after bubble collapse was assumed to have hyperbolic shape.

They have shown the height of the rising jet to be:

$$
h_{j}=\sqrt{\frac{2}{\left(\frac{g \rho_{f}}{\sigma}\right)-\left(\frac{1}{x y}\right)}} .
$$




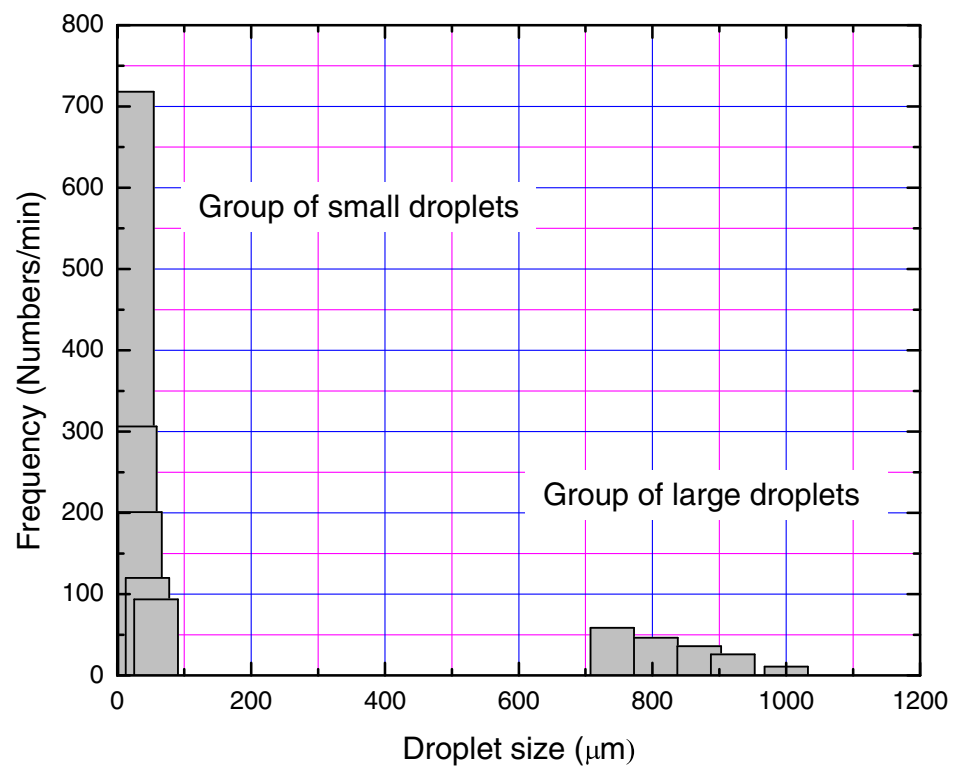

Figure 5. Size and frequency of film and jet droplets (taken from Newitt et al 1954).

They have further estimated the velocity of the rising jet and is given by:

$$
V=\frac{3}{2} \frac{\left(P_{o}+\frac{4 \gamma}{D}\right)}{D \rho_{f}} t .
$$

Newitt et al (1954) assumed $t$ to be of the order of 0.00003 seconds and found that the observed velocity of jet droplets agrees well with that given by Equation (9). They considered the criterion of breakup of cylindrical liquid column into drops when its length becomes greater than the circumference. Further, they employed the recommendation of Weber upon the favourable wave length at which such breakup occurs. As a result, they have shown that the jet droplet diameter is related with the jet diameter by the following equation:

$$
D=1.89 D_{j}
$$

Experimental observations by Newitt et al (1954) for jet droplet diameter were close to those given by the above equations. Authors also developed equations of drop ballistics based on the force balance for droplets. The maximum height attained by the droplets was found to be predicted accurately by the proposed theoretical procedure.

Garner et al (1954) measured the droplet size distribution (using MgO coated slides) inside an evaporator apparatus for different steam rates. They measured the entrainment carried at the exit of equipment by chemical analysis of salt (potassium nitrate) premixed in water. They also investigated the effect of bubble size on the production of various sizes of droplets. For this purpose, they used air-water system.

Figure 6 shows the experimental observations of Garner et al (1954) for the frequency of generation of various sized droplets. The results appear to be consistent with the findings of Newitt et al (1954) thus underlining the fact of existence of very high number of small droplets 


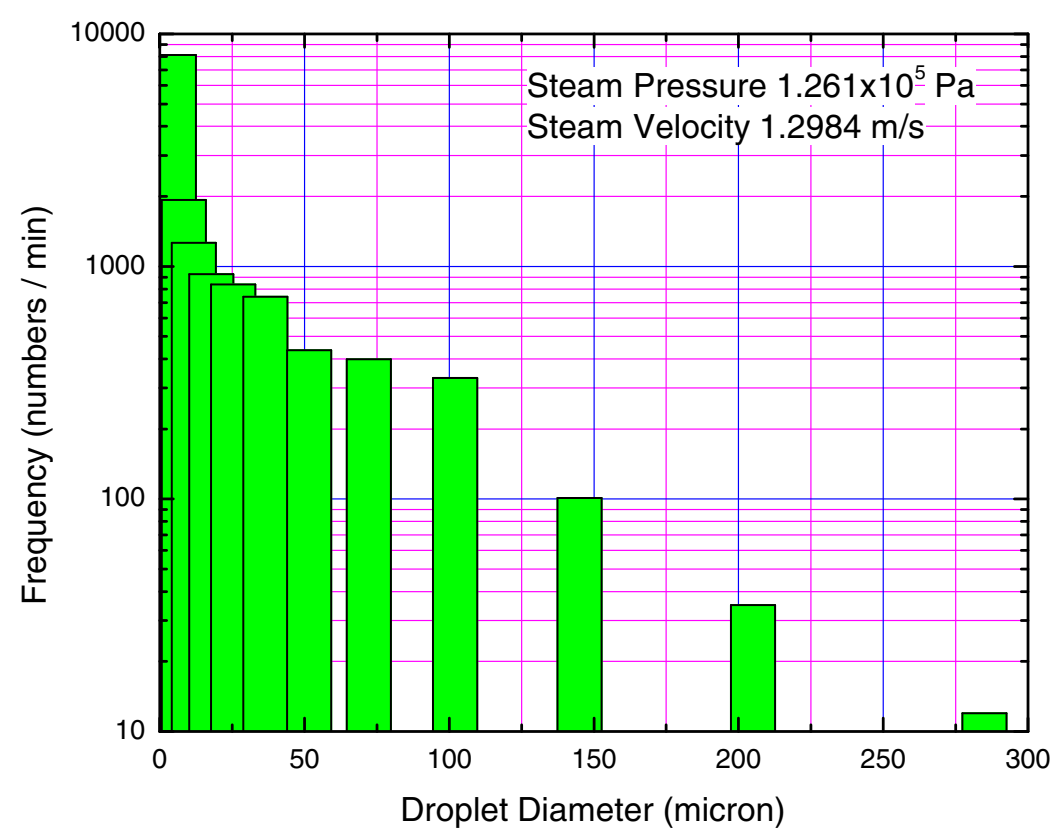

Figure 6. Frequency of droplets of different sizes (taken from Garner et al 1954).

above the separation surface. More than $95 \%$ of droplets were below $20 \mu$ size. Figure 7 shows the entrainment as a function of droplet diameter. It is interesting to see that, in spite of a decrease in number with an increase in size, the mass fraction of entrainment was found to increase with increase in the droplet diameter as the liquid volume is proportional to $D^{3}$. The larger droplets were obviously present closer to separation interface and thus entrainment was found to be high

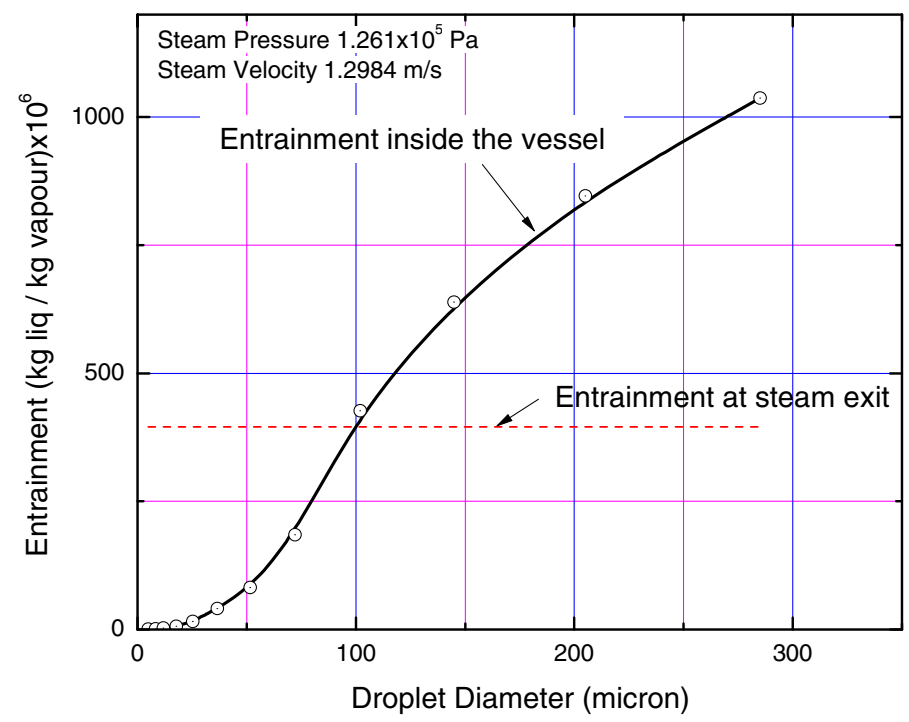

Figure 7. Entrainment as a function of droplet diameter (taken from Garner et al 1954). 


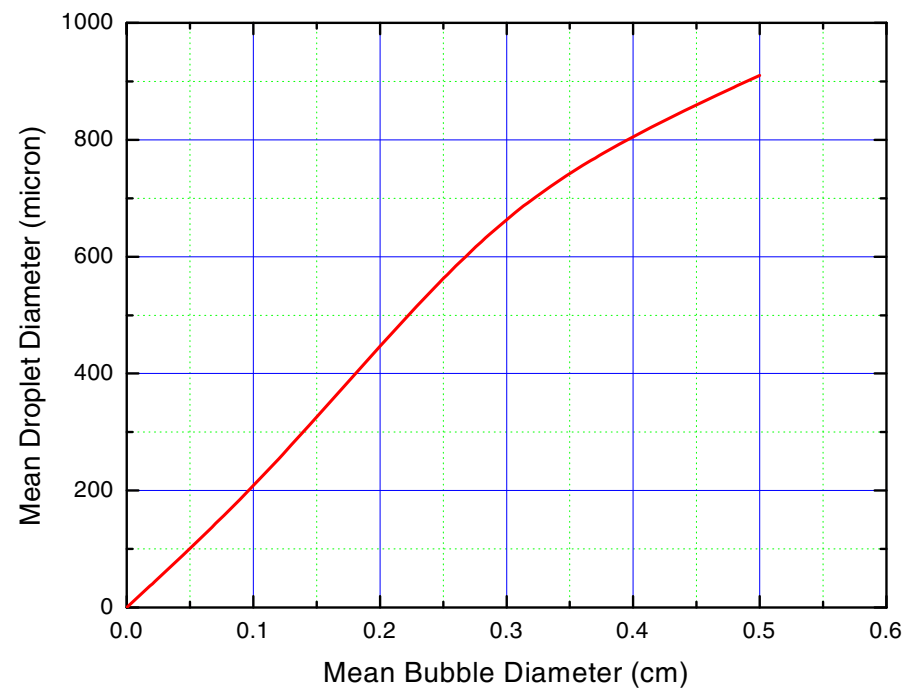

Figure 8. Relationship between bubble diameter and droplet size (taken from Garner et al 1954).

near the surface. Away from the surface up to exit, as expected the entrainment was found to decrease as the mean droplet size decreases. Garner et al (1954) found that $95 \%$ of the droplets entrained in the vapour space were having size less than $20 \mu$. However as the size of droplets is small, the total entrainment caused by these droplets is small. Garner et al (1954) found that droplets above $100 \mu$ would be the major contributor to entrainment.

Lowering the liquid level inside the evaporator was found to reduce the entrainment. Experimental results point out that the entrainment rises quite fast beyond a certain steam rate. At high vapour rate the liquid droplet flux at the separation interface rises due to agitation of boiling water. This results in to high entrainment rates. Author also noted that in the presence of foaming agent, a foam blanket is formed at the separation interface which acts as a viscous layer eliminating the droplets formed by splashing and thus reducing entrainment.

Garner et al (1954) investigated factors that influence size of droplets formed in the air-water system. They measured mean bubble diameter and the droplet size distribution at a distance of $10 \mathrm{~mm}$ above the free surface. The important experimental observations are shown in figures 8 and 9.

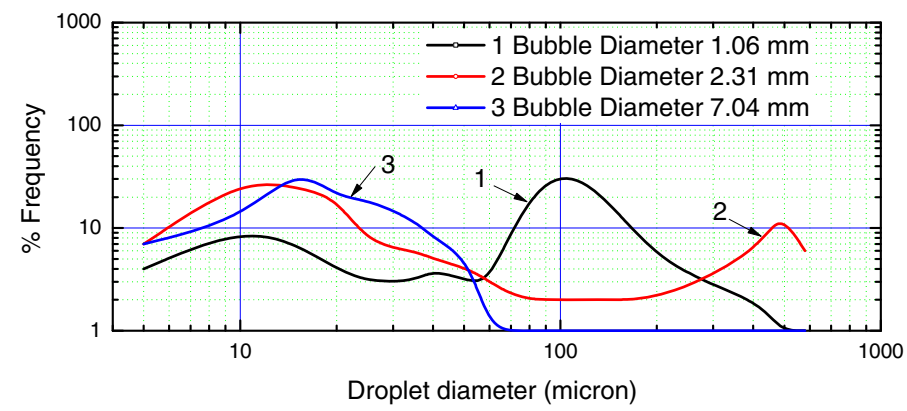

Figure 9. Effect of bubble diameter on frequency of droplet production (taken from Garner et al 1954). 


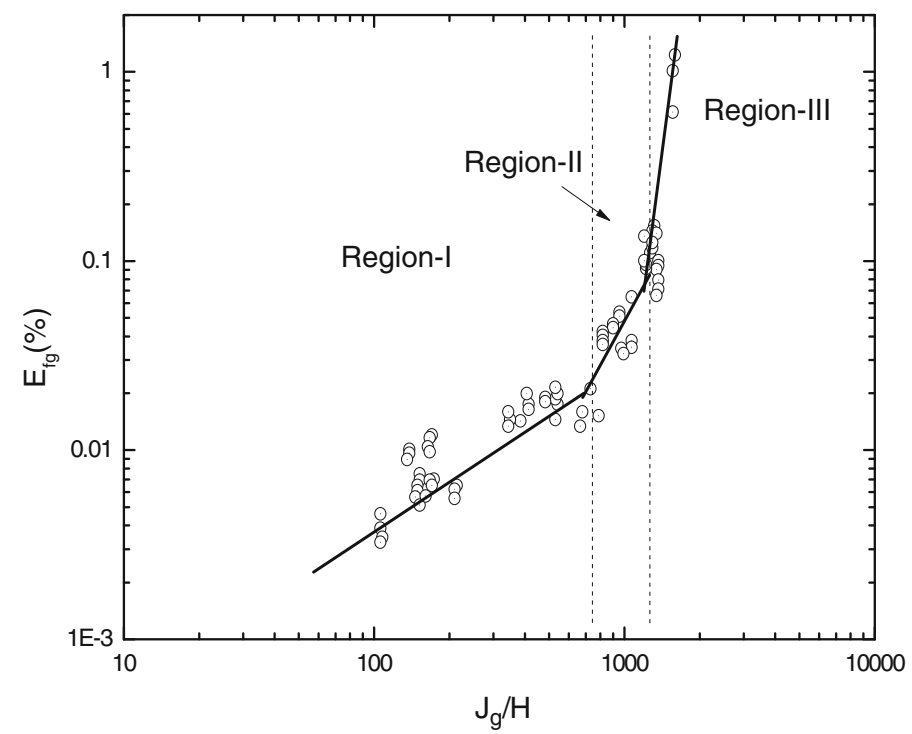

Figure 10. Entrainment as function of superficial velocity of steam and height of the vapour dome (taken from Sterman 1958).

The mean droplet diameter increased initially but after bubble size of $5 \mathrm{~mm}$ the mechanism of formation of jet droplet was found to be ineffective to produce droplets. For larger bubbles the mean droplet diameter dropped drastically (below $25 \mu$ ) and it was observed to be formed by the fragmentation of the liquid film. Thus Garner et al (1954) systematically provided insights into the droplet production mechanisms, their size distribution and influencing factors such as bubble diameter. Further, they have indicated the necessity of extensive experimentation to study the entrainment particularly at high pressures and establish factors affecting the droplet sizes, their projection velocities, resistance offered in the steam space, etc.

Figure 10 shows the entrainment measured at 185 atmosphere pressure. Different regions can be seen depending on the value of $J_{g} / h$. Experimental data can be approximated by power law, $E_{f g}=c\left(J_{g} / h\right)^{n}$ where $c$ and $n$ have different values for different regions. The three different regions can be explained with various scenarios shown in figure 11. Consider a vessel with initial liquid height $H_{L}$, and the gas/vapour flux in gradually increased. The two-phase mixture expands and separation interface height increases. At low gas flux values, the exit entrainment
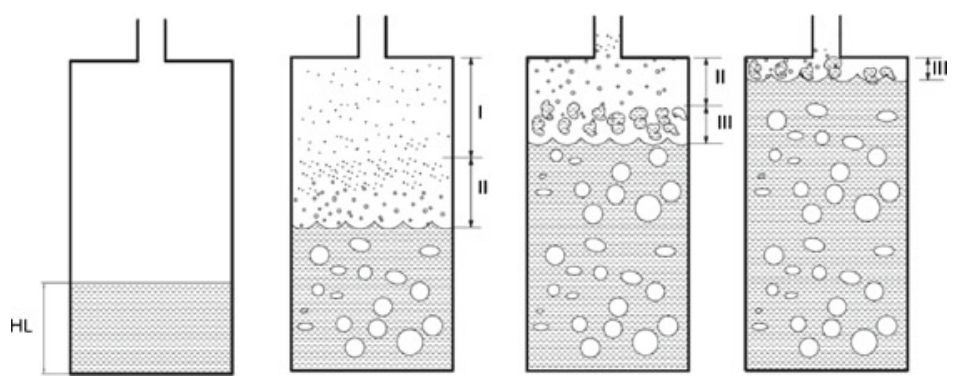

Figure 11. Mechanisms of entrainment. 
consists of small droplets which are carried along the vapour flow. Larger droplets fall back into the liquid. Hence resulting entrainment is small quantitatively. This is regarded as regionI, where exponent of $J_{g} / h$ is close to unity. As the gas flux increases the mixture height also increases reducing the vapour space above the separation interface. The large sized droplets are also reaching up to the exit and are carried out of the equipment. This shows increase in entrainment and change of slope of the curve. This is regarded as region-II, where exponent of $J_{g} / h$ is about 3.0 to 4.0. At very high gas flux the height of vapour space further reduces and the separation interface reaches very close to the exit. In this case the entrainment rises sharply. The separation interface is highly agitated at high gas flux and the large liquid fragments and droplets are carried out of equipment along the gas. This is regarded as region-III, where exponent of $J_{g} / h$ is about 7.0 to 20.0 .

Aiba \& Yamada (1959) studied the entrainment phenomena experimentally with air-water mixture. Experiments were carried out in a vertical cylinder filled with water and air injection at the bottom with a sparger pipe. Measurement of droplet sizes was carried out at different elevations using glass plates and cascade impactor. From cumulative volume fraction measured at various heights the mean droplet diameters were found out at the corresponding height. Using the equation of motion from force balance for the mean droplet size, projection velocity at separation interface was back calculated. This can be considered to be the most important result of the experiments. Garner et al (1954) and Newitt et al (1954) did not obtain the droplet ejection velocities. Aiba \& Yamada (1959) made estimation on droplet ejection velocities for the first time. The mean velocity of ejection was shown to decrease with increasing mean droplet size. However, reliable information on the droplet ejection velocity is still needed for case of high pressure systems involving steam-water mixture. Rozen et al (1970) analyzed the carryover in a conventional bubble type evaporators and bubble columns. Experimental measurements included the droplet collection on the plate coated with carbon layer at different elevations in the equipment. The droplet size distribution was found to be normal and distribution parameter was found to be independent of height. Only the mean droplet diameter was observed to change with respect to height. The total carryover out of the equipment was estimated from the samples and measuring the content of soluble salt (sodium chloride). The carryover has been described as a function of separator height using the following power-law equation proposed by Sterman (1958):

$$
E_{f g}=c\left(J_{g}\right)^{n} \text {. }
$$

On the basis of available experimental data Rozen et al (1970) found two regions. In the first region of relatively low vapour velocity the carryover was found to depend weakly on the vapour velocity. The value of power is unity in this region. In the second region of high velocities, the value of power $n=4.5$ to 5.5. They attributed that the exponent is a result of atomization laws, dispersion and distribution of drop dimensions. First region indicates different mechanism of droplet formation i.e., bubble breakdown.

Mochizuki \& Hirao (1993) performed experiments on the pilot model of the steam separator for the Advanced Thermal Reactor (ATR) which is a boiling light water nuclear reactor. The experiments were performed with air-water mixture at atmospheric conditions and the entrainment was measured using tracer salt and with iso-kinetic probe which is generally used in aerosol measurements. Based on the basic data separation efficiency was found to be acceptable for reactor geometry.

Spiel (1994) studied the size distribution of jet drops produced by the bursting of bubbles of dimensions ranging from 350 to $1500 \mu \mathrm{m}$ at sea as well as fresh water surfaces. He found 
no difference between the two results. He showed that different sizes of drops are produced by single bubble collapse. Further, probability of formation of first drop is the highest. In addition, the probability of formation decreases for second, third and subsequent droplets. Spiel (1994) studied the jet droplets produced by the bubble collapse for different bubble diameters. The size distribution for the drops other than the first top drop was found to be bimodal. The top drop radius was found to be dependent on bubble radius by:

$$
R_{d}=0.0377 R_{b}^{1.208}
$$

Cosandy et al (2001) analyzed the problem of radio-nuclide entrainment in the reactor containment in case of severe accident where the core melt is covered with the pool of water. Bubbling at the surface of water would transport radionuclides by carryover phenomenon. They performed measurements of carryover at different elevations in the containment model by sampling and measuring the soluble salt concentration (KI, NaI and CsI) and also of solid tracer (alumina).

Koch et al (2000) studied the carryover of radionuclides from bubbling pool of water in case of severe accident. Koch et al (2000) modelled droplet formation at bubbling surface using empirical correlations. The droplet formation at bubbling surface depends on the various mechanisms of formation. The droplets generated from the rising jet disintegration are called as jet droplets. Droplets formed due to disintegration of bubble liquid film are called as film droplets. When the steam flow rates are high, steam jets out at bubbling surface and shearing the liquid into droplets. This are called as churn flow droplets. Authors used correlations for the film droplets and churn flow droplets. The jet droplets were modelled using numerical method, solving the Navier-Stokes equation on Eulerian grid. The computer module RECOM (Voßnacke et al 1997) based on above models was validated against experimental data from KAREX facility (Minges et al 1998). Good agreement was found by authors between theoretical and experimental results.

Gunther et al (2003) carried out experiments on the droplet production rate at a water surface with the presence of single and multiple air bubbles of same dimensions ( $3 \mathrm{~mm}$ diameter). Effect on the droplet size distribution because of the presence of multiple bubbles was found to be important as the erstwhile correlations were pointed out to be based on the data from collapse of a single bubble. The measurements were carried out using Phase Doppler Analyzer (PDA). It was found that the number of droplets produced by the disintegration of fluid film upon the collapse of bubble is one magnitude higher than the droplets produced by the momentum leading to rise of jet at the centre and its necking. Jet drops from single bubble had higher diameter than in case of multiple bubbles. Coalescence of small bubbles creates larger bubbles of dimensions $4 \mathrm{~mm}$ in diameter and the droplets produced by the film disintegration was found to be dominant. Due to insufficient gas velocities all these were found to be re-entrained in the pool.

Kim \& No (2005) studied liquid entrainment in the case of a vessel of the depressurization system of Advanced Power Reactor (APR-1400). The liquid off take from the swelled two-phase mixture surface in vessel was studied with experiments on the model with air-water mixture. Entrainment was measured by directly noting the water collected at the end of discharge pipe. Isokinetic probes were also utilized to measure the entrainment fractions. They described the entrainment as a function of the distance between the break location and swell level of twophase mixture pool. As shown in figure 11, when the break is close to the level of mixture, the vapour accelerates through break due to sudden area change (Bernoulli Effect) and can carry large amount of liquid sheets splashing at the interface. While at lower mixture levels the droplets either may get carried away with flow or fall back into the vessel. Authors developed a new correlation for the liquid entrainment and the experimental data were found to be proportional to the seventh power of $J_{g}^{*} h^{*-1}$ and had higher values than other existing correlations. 


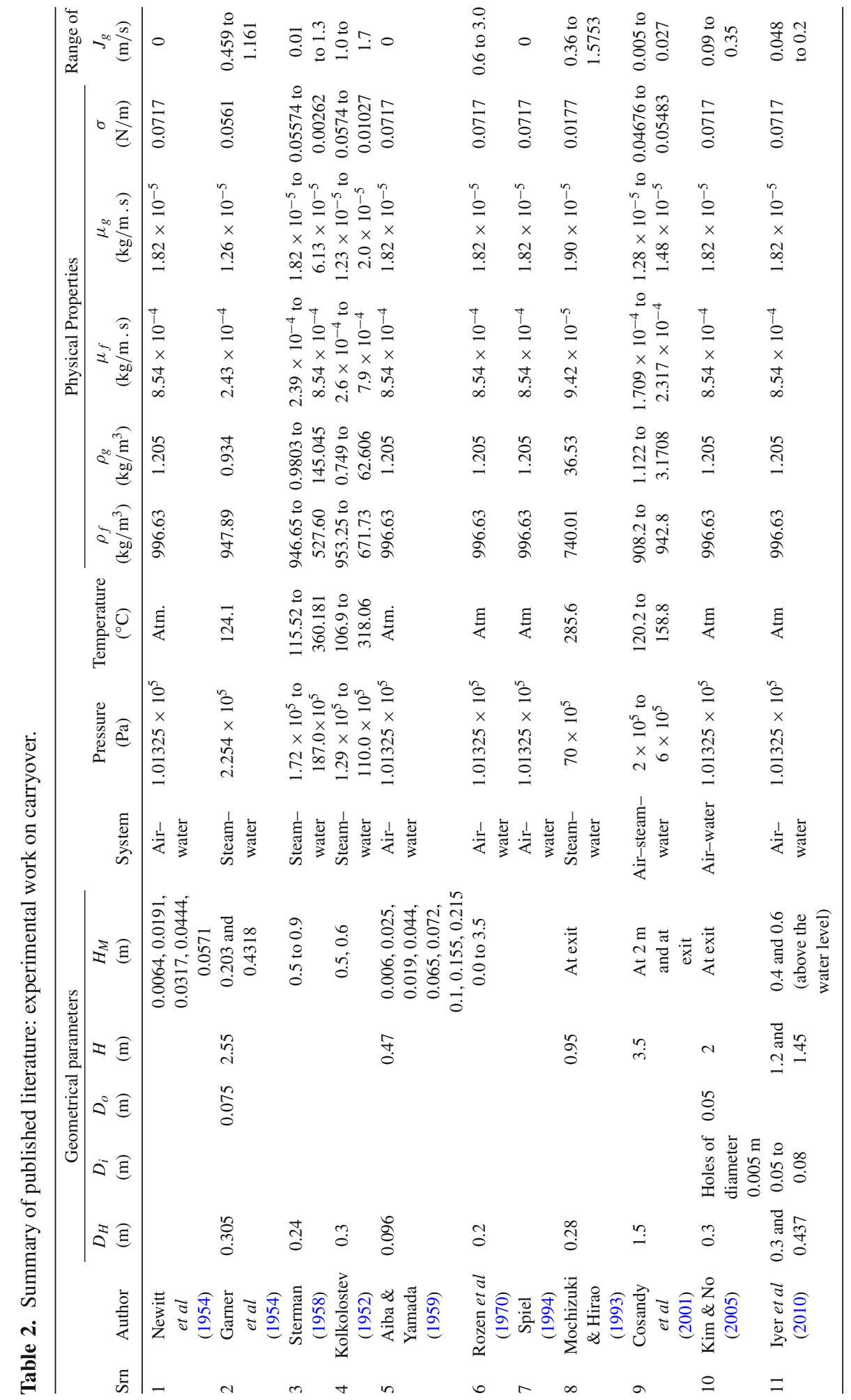


Iyer et al (2010) studied the effect of superficial gas velocity and the diameter ratio (of vessel to inlet pipe) on the carryover. The experiments were performed carried out at atmospheric conditions with air and water mixture. The carryover was measured with the help of tissue papers suspended above the separation interface and the collected amount of entrainment was weighed. Authors proposed a new correlation based on experimental data. The proposed correlation satisfies their experimental data within 375\%. The various experimental studies conducted on carryover are summarized in table 2.

\section{Empirical correlations}

Kruzhilin (1951) developed a correlation for the entrainment assuming that the droplets generated from the disintegration of a bubble dome contribute little to the entrainment. Further, he opinioned that the entrainment is mainly due to the droplets formed by the action of kinetic energy of vapour. The effect of drop disintegration/coalescence can also be neglected. Kruzhilin (1951) listed the parameters that affect the entrainment. These are $\rho_{g} J_{g}^{2}, \rho_{f}, \sigma, \mu$

Following dimensionless groups were defined:

$$
\begin{gathered}
\Pi_{1}=\frac{G_{f} \sigma g}{\left(\rho_{g} J_{g}^{2}\right)^{2} J_{g}} \sqrt{\frac{\rho_{f}}{\rho_{g}}}, \\
\Pi_{2}=\frac{\mu_{f}^{2} \rho_{g} J_{g}^{2}}{\sigma^{2} \rho_{f}}, \\
\Pi_{3}=\frac{\rho_{g} J_{g}^{2}}{g \rho_{f} H_{V}} \Rightarrow \text { Froude number, } \\
\Pi_{4}=\frac{\sigma}{g \rho_{f} H_{V}^{2}} \Rightarrow \text { Weber number. }
\end{gathered}
$$

From the definition of entrainment

$$
E_{f g}=\frac{\text { Flux of liquid phase }}{\text { Flux of vapour phase }}=\frac{G_{f}}{\rho_{g} J_{g}}=\frac{\rho_{f} J_{f}}{\rho_{g} J_{g}} .
$$

Neglecting the effect of droplet coalescence/disintegration, the Weber number was neglected from the dimensionless groups and thus the entrainment was related by the following correlation:

$$
E_{f g}=\left[\frac{\rho_{g} J_{g}^{4}}{\sigma g} \sqrt{\frac{\rho_{g}}{\rho_{f}}}\right] f\left(\frac{\mu_{f}^{2} \rho_{g} J_{g}^{2}}{\sigma^{2} \rho_{f}}, \frac{\rho_{g} J_{g}^{2}}{g \rho_{f} H_{V}}\right) .
$$

The function $f\left(\frac{\mu_{f}^{2} \rho_{g} J_{g}^{2}}{\sigma^{2} \rho_{f}}, \frac{\rho_{g} J_{g}^{2}}{g \rho_{f} H_{V}}\right)$ was assumed to be constant and thus

$$
E_{f g}=c_{1} \frac{\rho_{g} J_{g}^{4}}{\sigma g} \sqrt{\frac{\rho_{g}}{\rho_{f}}} .
$$

It must be noted that height of vapour dome $H_{V}$ has not been included in the above expression; hence it cannot predict entrainment for any general height of vapour dome. 
Panasenko (1959) attempted to obtain the correlation for the data at the transition point from region II to region III (i.e., region where droplets from bubble disintegration are dominant as compared with the region where highly agitated liquid splashes cause the major entrainment (see figure 11). Authors extended the important results of Kruzhilin (1951) and described the entrainment as:

$$
E_{f g}=c_{2}\left[\frac{\rho_{g}}{\sigma g} \sqrt{\frac{\rho_{g}}{\rho_{f}}} J_{g}^{4}\right] \Pi_{2}^{m} \Pi_{3}^{n} \Pi_{4}^{l},
$$

where $\Pi_{2}, \Pi_{3}$ and $\Pi_{4}$ are given by equation 14,15 and 16, respectively. The constants were obtained by fitting the experimental data by Sterman (1958) and given by the following equation:

$$
E_{f g}=1.96 \times 10^{7} \frac{\left(\rho_{g} g\right)^{0.48} \mu_{f}^{1.8} J_{g}^{1.96}}{g^{0.08}\left(\rho_{g} g\right)^{1.03} \sigma^{1.25} H_{V}^{1.18}} .
$$

Note that the above expression shows dependence on $H_{V}$ i.e., height of vapour space which can be given as

$$
H_{V}=H-H_{L}\left(\frac{\alpha}{1-\alpha}\right)
$$

where the pool void fraction $\alpha$, is given as:

$$
\alpha=0.67\left(\frac{J_{g}^{2}}{g \sqrt{\frac{\sigma}{g\left(\rho_{f}-\rho_{g}\right)}}}\right)^{1 / 3}\left(\frac{\rho_{f}-\rho_{g}}{\rho_{f}}\right)^{-1 / 3}\left(\frac{V_{f}}{V_{g}}\right)^{2 / 9}\left(\frac{D_{H}}{\sqrt{\frac{\sigma}{g\left(\rho_{f}-\rho_{g}\right)}}}\right)^{-1 / 6} .
$$

Sterman (1958) attempted to provide groups of dimensionless numbers based on the processes taking place during the droplet formation and their transport with steam. His attempt of arriving at number of dimensionless groups affecting the process was inspired by the fact that the earlier correlations were unable to resolve his experimental data and the procedure adopted by earlier work did not consider all the factors which are associated with the phenomenon of drop formation and their motion. He neglected the droplets formed due to disintegration of bubble dome, instead considered the droplet formation due to kinetic energy of vapour that works against surface tension and friction with liquid film. He described the process using energy balance during drop formation as per the following equation:

Rate of change of kinetic energy $=$ Rate of change of (surface energy + heat energy + potential energy)

$$
D_{H}\left(\frac{m_{e f} V_{f}^{2}}{2}+\frac{m_{e g} V_{g}^{2}}{2}\right)=s . d s+\frac{g}{A} h_{f g} d m_{e f}+g\left(m_{e f}+m_{e g}\right) d h .
$$

The force balance for a droplet can be described as

$$
c_{1} \rho_{f} D^{3} \frac{d V_{d}}{d t}-c_{2} C_{D} D^{2} \frac{\rho_{f} g\left(V_{d}-V_{g}\right)}{2}=c_{3} D g \rho_{f},
$$

and the process of evaporation of drops can be described using the following Laplace and Clausius-Clapeyron equation, respectively,

$$
\Delta P=P_{1}-P_{o}=\frac{4 \sigma}{D} \frac{\rho_{f}}{\rho_{f}-\rho_{g}}
$$




$$
\frac{d T_{s}}{d p}=\frac{A T_{s}\left(\rho_{f}-\rho_{g}\right)}{h_{f g} \rho_{f} \rho_{g}} .
$$

From Eq. (24) to (27) author developed a similarity criteria in which parameters from equation of motion of two-phase (below the steam space) were also included

$$
E_{f g}=f\left\{w, \operatorname{Re}_{g}, E u, \frac{\rho_{g} J_{g}^{2} D}{g \sigma}, \frac{J_{g}^{2}}{g H_{V}}, \frac{\Delta P}{\frac{\sigma}{D}}, \frac{A \Delta P}{h_{f g} \rho_{g}} \frac{T_{s}}{\Delta T}, \alpha, \frac{H_{V}}{D}, \frac{V_{d}}{J_{g}}, \frac{\rho_{g}}{\rho_{f}}\right\} .
$$

After neglecting the terms related to droplet evaporation and droplet diameter and droplet velocity, the similarity criteria can be written as:

$$
E_{f g}=f\left\{\frac{g\left(\sqrt{\frac{\sigma}{\rho_{g}}}\right)^{3}}{V_{g}^{2}}, \frac{J_{g}^{2}}{g H_{V}}, \frac{A \sqrt{\frac{\sigma}{\rho_{g}}}}{h_{f g}}, \frac{H_{V}}{\sqrt{\frac{\sigma}{\rho_{g}}}}, \alpha, \frac{\rho_{g}}{\rho_{f}}\right\} .
$$

In the above criterion $H_{V} / \sqrt{\sigma / \rho_{g}}$ was found to have no significant effect when $H_{V}$ is small hence it was neglected.

Also the self-evaporation of droplet was neglected. Thus the term $\left(A \sqrt{\sigma / \rho_{g}}\right) / h_{f g}$ was neglected.

Finally, the entrainment was expressed in terms of dryness fraction of steam which is defined as:

$$
E_{f g}=1-w=f\left\{\frac{g\left(\sqrt{\frac{\sigma}{\rho_{g}}}\right)^{3}}{v}, \frac{J_{g}^{2}}{g H_{V}}, \alpha, \frac{\rho_{g}}{\rho_{f}-\rho_{g}}\right\} .
$$

Sterman (1958) analyzed the experimental data of Kolkolostev (1952) and Sterman (1958). He proposed following correlation:

$$
w=2.75 \times 10^{8} \frac{\left(\frac{J_{g}^{2}}{\alpha g H_{V}}\right)^{2.3}}{\left[\frac{g\left(\frac{\sigma}{g\left(\rho_{f}-\rho_{g}\right)}\right)^{3 / 2}}{v^{2}}\right]^{1.1}\left(\frac{\rho_{g}}{\rho_{f}-\rho_{g}}\right)^{0.25}}
$$

In the above correlation the knowledge of void fraction $(\alpha)$ and height of vapour dome $\left(H_{V}\right)$ is needed. The correlations have been proposed for $(\alpha)$ and $\left(H_{V}\right)$.

$$
\alpha=0.26\left(\frac{J_{g}^{2}}{g \sqrt{\frac{\sigma}{\rho_{f}-\rho_{g}}}}\right)^{0.4}\left(\frac{\rho_{g}}{\rho_{f}-\rho_{g}}\right)^{0.12}
$$

where

$$
\frac{D}{\sqrt{\frac{\sigma}{\rho_{f}-\rho_{g}}}}\left(\frac{\rho_{g}}{\rho_{f}-\rho_{g}}\right)^{-0.2} \geq 260
$$




$$
H_{V}=H-\left(\frac{\alpha}{1-\alpha}\right) H_{L}
$$

Combining Eq. (27) to (29), the final correlation was proposed as follows:

$$
w=6.1 \times 10^{9} \frac{\left(\frac{J_{g}^{2}}{g H_{V}}\right)^{1.38}}{A r^{1.1}}\left(\sqrt{\frac{\sigma}{\rho_{f}-\rho_{g}}}\right),
$$

where

$$
A r=\frac{g\left(\sqrt{\frac{\sigma}{\rho_{f}-\rho_{g}}}\right)^{3}}{V_{g}^{2}} \frac{\rho_{f}-\rho_{g}}{\rho_{g}} .
$$

The above correlation indicates that the entrainment also depends on hydrodynamics of twophase flow below the separation interface.

Ishii \& Kataoka (1984) developed a mechanistic model by combining the physics of phenomenon (droplet formation, size and velocity distribution, droplet motion, etc.) known from the observations and various experimental data (entrainment data with air-water and steam-water) reported by Kolkolostev (1952), Garner et al (1954), Sterman (1958) and Rozen et al (1970). The authors thought that the phenomenon of droplet generation can be treated statistically by introducing the distribution functions for size and velocity. The following relations were found to hold for size distribution function $f\left(D, J_{g}\right)$ and velocity distribution function $g\left(V_{i}, D, J_{g}\right)$ :

$$
\begin{aligned}
& \int_{0}^{\infty} f\left(D, J_{g}\right) d D=1, \\
& \int_{0}^{\infty} g\left(V_{i}, D, J_{g}\right) d V_{i}=1 .
\end{aligned}
$$

Let $V_{h}$ be the velocity required for droplet to attain height ' $h$ ', then the entrainment can be given as,

$$
E_{f g}\left(h, J_{g}\right)=\frac{\varepsilon\left(\dot{J}_{g}\right)}{\rho_{g} J_{g}} \int_{0}^{\infty} \int_{V_{h}\left(D, J_{g}, h\right)}^{\infty} g\left(V_{i}, D, J_{g}\right) f\left(D, J_{g}\right) d V_{i} d D,
$$

where $\dot{\varepsilon}\left(J_{g}\right) / \rho_{g} J_{g}$ is the entrainment rate at the interface.

The droplets can be categorized into two groups, i.e.,

1. $D<D_{c}$, where $D_{c}$ is critical diameter of droplet for which the terminal velocity is equal to vapour velocity. These droplets are always carried in the flow.

2. $D>D_{c}$, these rise to certain height and then fall back.

Introducing a term representing the entrainment at the surface as:

$$
E_{0}\left(D, J_{g}\right)=\frac{\varepsilon\left(\dot{J}_{g}\right)}{\rho_{g} J_{g}} \int_{0}^{D} f\left(D, J_{g}\right) d D
$$


Garner et al (1954) measured this quantity, which can be expressed as

$$
E_{0}\left(D, J_{g}\right) \propto\left(J_{g} D\right)^{1.5} .
$$

After fitting experimental data, the following correlation was obtained:

$$
E_{0}\left(D, J_{g}\right)=0.3975\left(J_{g}^{*} D^{*}\right)^{1.5}
$$

where

$$
J_{g}^{*}=\frac{J_{g}}{\left(\frac{\sigma g \Delta \rho}{\rho_{g}^{2}}\right)^{1 / 4}} \quad \text { and } \quad D^{*}=\frac{D}{\sqrt{\sigma / g \Delta \rho}} .
$$

Incorporating the effect of pressure in Equation (38), we get:

$$
E_{0}\left(D, J_{g}\right)=\frac{\varepsilon\left(\dot{J}_{g}\right)}{\rho_{g} J_{g}} \int_{0}^{D} f\left(D, J_{g}\right) d D=2.48 \times 10^{-4}\left(\frac{\rho_{g}}{\Delta \rho}\right)^{-1.0}\left(J_{g}^{*} D^{*}\right)^{1.5}
$$

Differentiating above equation with respect to $D$,

$$
\sqrt{\frac{\sigma}{g \Delta \rho}} \frac{\varepsilon\left(\dot{J}_{g}\right)}{\rho_{g} J_{g}} f\left(D, J_{g}\right)=3.72 \times 10^{-4}\left(\frac{\rho_{g}}{\Delta \rho}\right)^{-1.0}\left(J_{g}^{*} D^{*}\right)^{1.5} .
$$

This equation gives the relationship between the entrainment at the interface and the droplet size distribution in terms of steam superficial velocity and the droplet diameter.

It has been known that the droplet formation mechanism depends on the pool hydrodynamics. The gas velocities required for bubbly flow in the pool is given by the drift flux model (Ishii 1977) given by the following equation:

$$
J_{g}^{*}=0.325 \sqrt{\frac{\rho_{g}}{\rho_{f}}} .
$$

For bubbly flow the value of $J_{g}$ required is very small. Therefore, Ishii \& Kataoka (1984) stated that, in equipments of practical interest, seldom bubbly flow would exist. Authors envisaged that the churn turbulent flow may be the most dominant flow regime in bubbling pool. Thus the mechanism of droplet generation due to bubble burst (film droplets and jet droplets) was not considered in the formulation. The authors alternatively proposed a mechanism as schematically shown in figure 12.

The force balance for a liquid film being sheared by the gas is written as:

$$
\rho_{f} D \frac{d V_{f}}{d t}=\tau_{i}-\Delta \rho g D
$$

where

$$
\tau_{i}=f_{i} \frac{1}{2} \rho_{g} V_{g}^{2} .
$$

Neglecting the gravity term, they obtained:

$$
\frac{d V_{f}}{\frac{1}{2} \frac{\rho_{g}}{\rho_{f}} \frac{1}{D} f_{i} V_{g}^{2}}=d t=\frac{d z}{V_{f}} .
$$



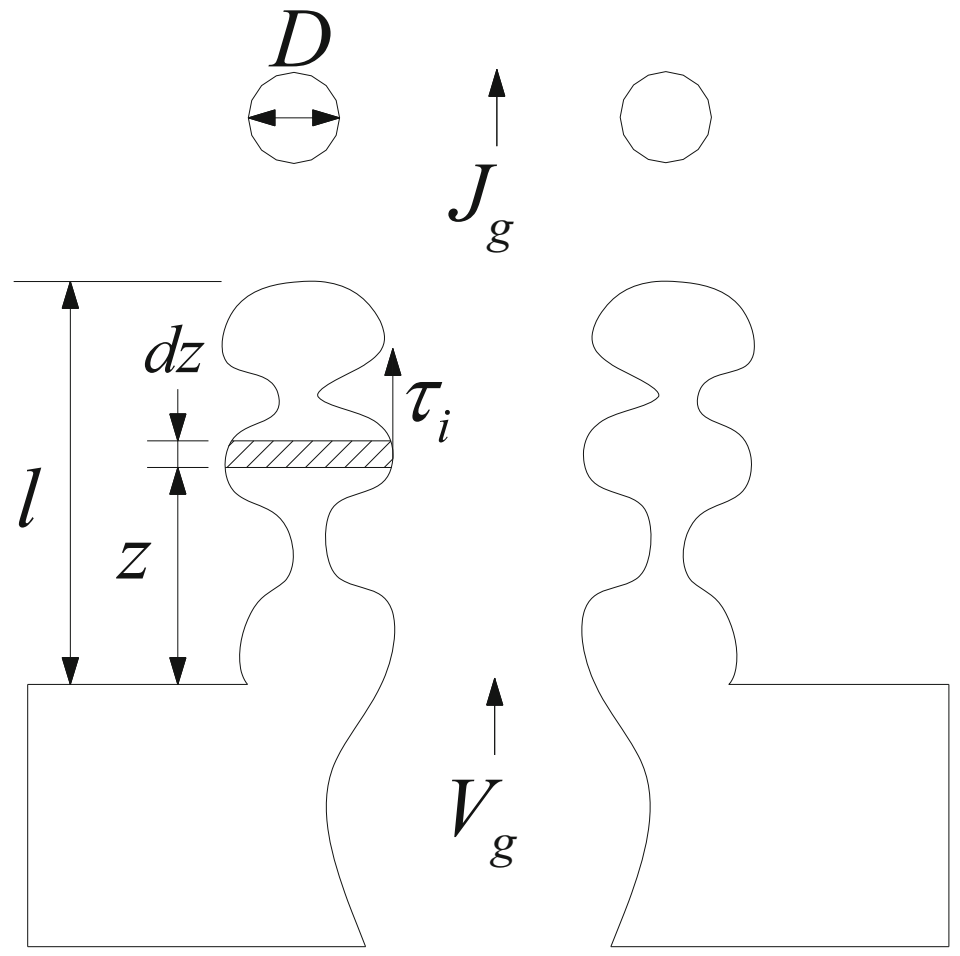

Figure 12. Formation of film droplets by shear (taken from Ishii \& Kataoka 1984).

Integrating the above equation and putting $\left(V_{f}\right)_{z=l}=V_{i}$, we get:

$$
\frac{1}{2} \rho_{f} V_{i}^{2} D=\frac{1}{2} f_{i} \rho_{g} V_{g}^{2} l \text {. }
$$

Above equation shows that the kinetic energy of droplet is equal to the work exerted on the element by the gas flow. In addition, authors made assumptions that $f_{i} \approx C_{D} \approx \operatorname{Re}_{D}^{-0.5}$ and $l \approx D$, and obtained the following relationship:

$$
V_{i}^{*} \sim J_{g}^{* 3 / 4} \alpha^{-3 / 4} N_{\mu g}^{1 / 4} D^{*-1 / 4}\left(\frac{\rho_{g}}{\rho_{f}}\right)^{1 / 2},
$$

The gas velocity is related to the pool void fraction by:

$$
\begin{gathered}
V_{g}=\frac{J_{g}}{\alpha}, \\
V_{i}^{*} \sim V_{g}^{* 3 / 4} N_{\mu g}^{1 / 4} D^{*-1 / 4}\left(\frac{\rho_{g}}{\rho_{f}}\right)^{1 / 2},
\end{gathered}
$$

where

$$
N_{\mu g}=\frac{\mu_{g}}{\left(\rho_{g} \sigma \sqrt{\frac{\sigma}{g \Delta \rho}}\right)^{1 / 2}},
$$




$$
\begin{aligned}
& V_{i}^{*}=\frac{V_{i}}{\left(\frac{\sigma g \Delta \rho}{\rho_{g}^{2}}\right)^{1 / 4}}, \\
& V_{g}^{*}=\frac{V_{g}}{\left(\frac{\sigma g \Delta \rho}{\rho_{g}^{2}}\right)^{1 / 4}} .
\end{aligned}
$$

Ishii \& Kataoka (1984) proposed the following correlation for the void fraction as:

$$
\alpha \sim J_{g}^{* 2 / 3} D_{H}^{*-0.28}\left(\frac{\rho_{g}}{\Delta \rho}\right)^{-0.153}
$$

Therefore,

$$
V_{i}^{*}=C J_{g}^{* 1 / 4} N_{\mu g}^{1 / 4} D^{*-1 / 4}\left(\frac{\rho_{g}}{\rho_{f}}\right)^{1 / 2} D_{H}^{*-0.21}\left(\frac{\rho_{g}}{\Delta \rho}\right)^{-0.11} .
$$

The velocity distribution for a given size of droplet is neglected and instead it is considered to have average velocity for the given drop diameter. Therefore, it can be expressed with delta function as:

$$
g\left(V_{i}, D, J_{g}\right) d V_{i}=\delta\left(V_{i}^{*}-\overline{V_{i}^{*}}\right) d V_{i}^{*} .
$$

Ishii \& Kataoka (1984) also proposed a procedure for determining maximum height attained by the droplet. The force balance for the droplet can be written as:

$$
\frac{d V}{d t}=\frac{-\Delta \rho}{\rho_{f}} g-\frac{3}{4} C_{D} \frac{1}{D} \frac{\rho_{g}}{\rho_{f}}\left(V-V_{g}\right)\left|V-V_{g}\right| .
$$

Above equation was solved for various drop diameters and the following approximate solution was obtained for the velocity required by a droplet to reach height ' $h$ ',

$$
\begin{gathered}
V_{h}=0 \text { for } D<D_{c}, \\
V_{h}=\sqrt{2 g h\left(\frac{\rho_{g}}{\rho_{f}}\right)} \text { for } D \geq D_{c} .
\end{gathered}
$$

The entrainment now can be expressed as follows

$$
\begin{aligned}
E_{f g}\left(h, J_{g}\right)= & \int_{0}^{\infty} \int_{V_{h}\left(D, J_{g}, h\right)}^{\infty} \delta\left(V_{i}^{*}-\overline{V_{i}^{*}}\right) \times 3.72 \times 10^{-4} \\
& \times\left(\frac{\rho_{g}}{\Delta \rho}\right)^{-1.0} J_{g}^{* 1.5} D^{* 0.5} d V_{i}^{*} d D^{*}
\end{aligned}
$$

Integration of Eq. (60) shows three distinct regions for the value of $h$ as shown in figure 13.

Final set of correlations are as follows,

- Near surface region

$$
E_{f g}\left(h, J_{g}\right)=4.84 \times 10^{-3}\left(\frac{\rho_{g}}{\Delta \rho}\right)^{-1.0} \text { and } D_{\max }^{*}=7.24 J_{g}^{*-1} \text {. }
$$




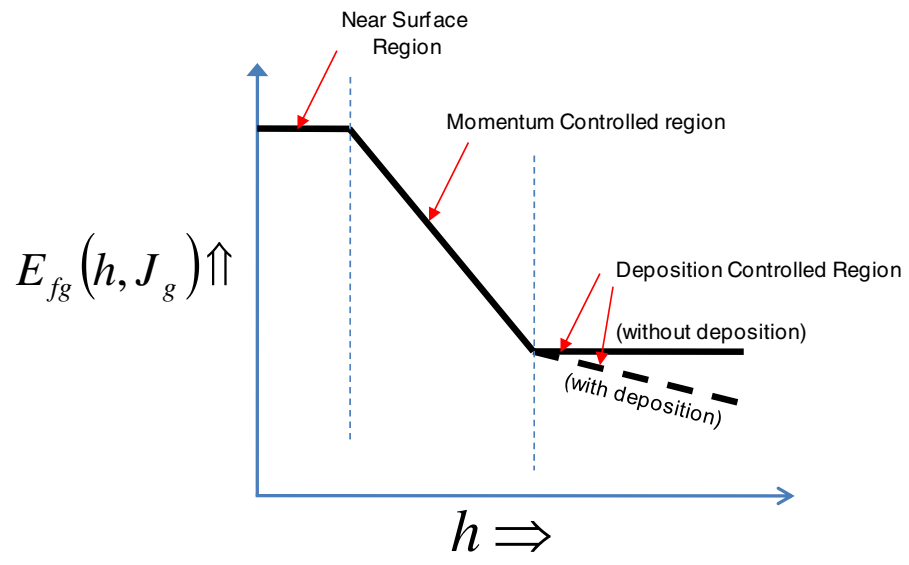

Figure 13. Regions of entrainment above the separation interface (taken from Ishii \& Kataoka 1984).

\section{- Deposition controlled region}

$$
E_{f g}\left(h, J_{g}\right)=7.13 \times 10^{-4} J_{g}^{* 3} N_{\mu g}^{0.5}\left(\frac{\rho_{g}}{\Delta \rho}\right)^{-1.0} \exp \left(-0.205 \frac{h}{D_{H}}\right) .
$$

\section{- The momentum controlled region}

For momentum controlled region the correlation for entrainment is given for three gas flux regimes, high, intermediate and low gas flux.

\section{- Low gas flux regime}

$$
E_{f g}\left(h, J_{g}\right)=2.21 N_{\mu g}^{1.5} \times D_{H}^{* 1.25}\left(\frac{\rho_{g}}{\Delta \rho}\right)^{-0.31} J_{g}^{*} h^{*-1} .
$$

The transition to intermediate gas flux regime occurs at

$$
J_{g}^{*} h^{*-1}=6.39 \times 10^{-4} .
$$

\section{- Intermediate gas flux regime}

$$
E_{f g}\left(h, J_{g}\right)=5.42 \times 10^{6} J_{g}^{* 3} h^{*-3} N_{\mu g}^{1.5} \times D_{H}^{* 1.25}\left(\frac{\rho_{g}}{\Delta \rho}\right)^{-1.0} .
$$

\section{- High gas flux regime}

Eq. (61) holds good for the high flux regime.

The various correlations discussed above are compared for the experimental data available in literature.

Figure 14 shows prediction for experimental measurements by Garner et al (1954) for airwater system at atmospheric pressure. The three correlations given by Sterman (1958), Kruzhilin (1951) were developed based on the experiment in steam-water system. Correlation given Ishii \& Kataoka (1984) considers experimental data from air-water system also. For the case of Garner et al (1954), Ishii \& Kataoka (1984) gave better prediction compared to others, but the error is noticeably very high (400-1000\%). Figures 15 to 19 show the comparison for experimental data 


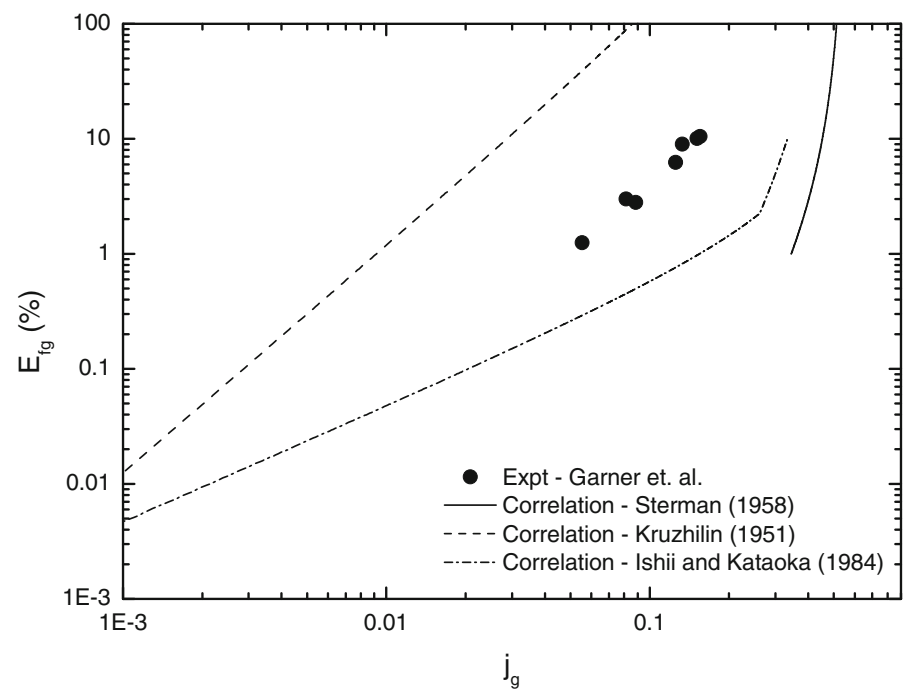

Figure 14. Prediction for Garner et al (1954).

on entrainment by Sterman (1958) and Kolkolostev (1952) for steam-water system at operating pressures of 1.27, 17.0, 36.0, 91.0 and 110.0 atmospheres respectively. The correlation by Ishii \& Kataoka (1984) predicts three different regions depending on the gas flux and height at which the entrainment is measured. However, the correlations by Kruzhilin (1951) and Sterman (1958) do not predict such three regions. The correlation by Ishii \& Kataoka (1984) is most accurate and can effectively predict the combined effect of height and gas flux on entrainment. Hence, it is recommended to be used for assessment of carryover in steam-water systems. It can be noted

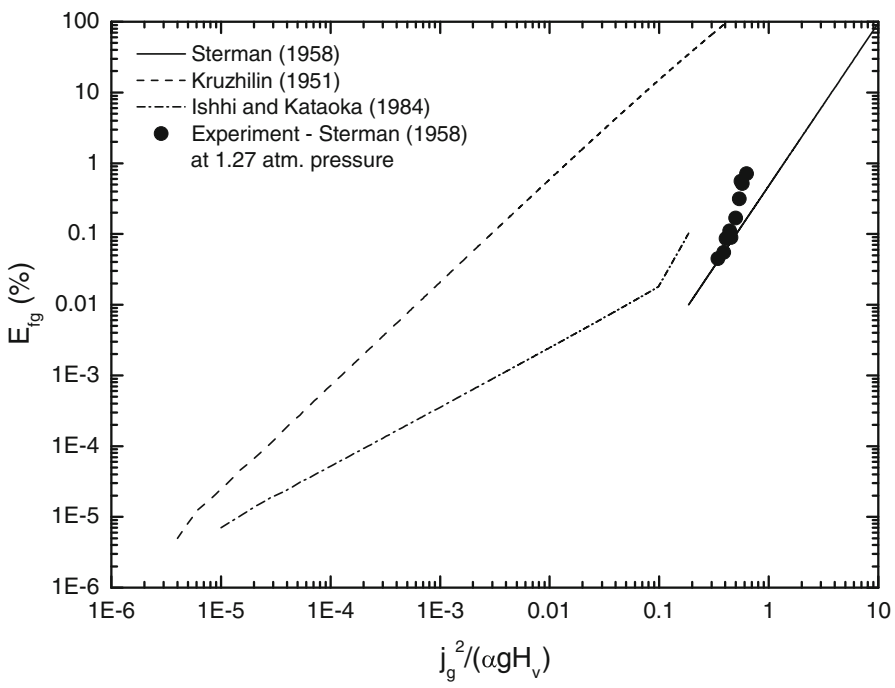

Figure 15. Prediction for Sterman (1958) for 1.27 atmospheric pressure. 


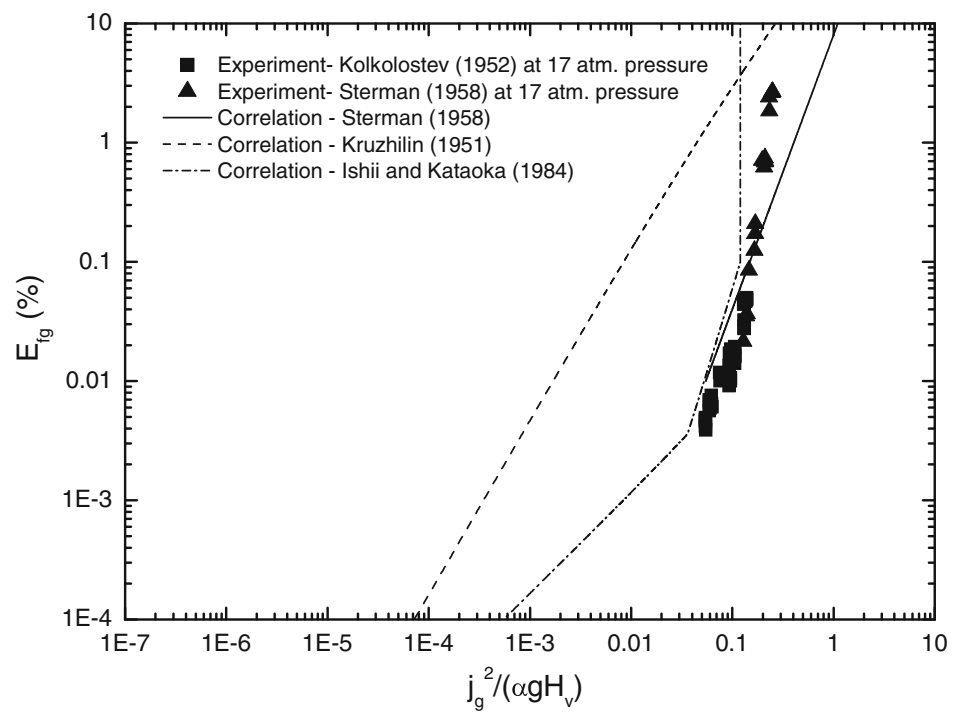

Figure 16. Prediction for Sterman (1958) and Kolkolostev (1952) at 17 atmospheric pressure.

that it is valid for equipments with constant flow area. This correlation was used in subsequent experimental work by Cosandy et al (2001) and Iyer et al (2010). These authors have found some disagreements between correlation and experimental observations, which is discussed further.

Cosandy et al (2001) analyzed the problem of radio-nuclide entrainment in the reactor containment in case of severe accident where the core melt is covered with the pool of water. Bubbling at the surface of water would transport radionuclides by carryover phenomenon. Authors predicted the measured carryover fractions using the correlation provided by Ishii \&

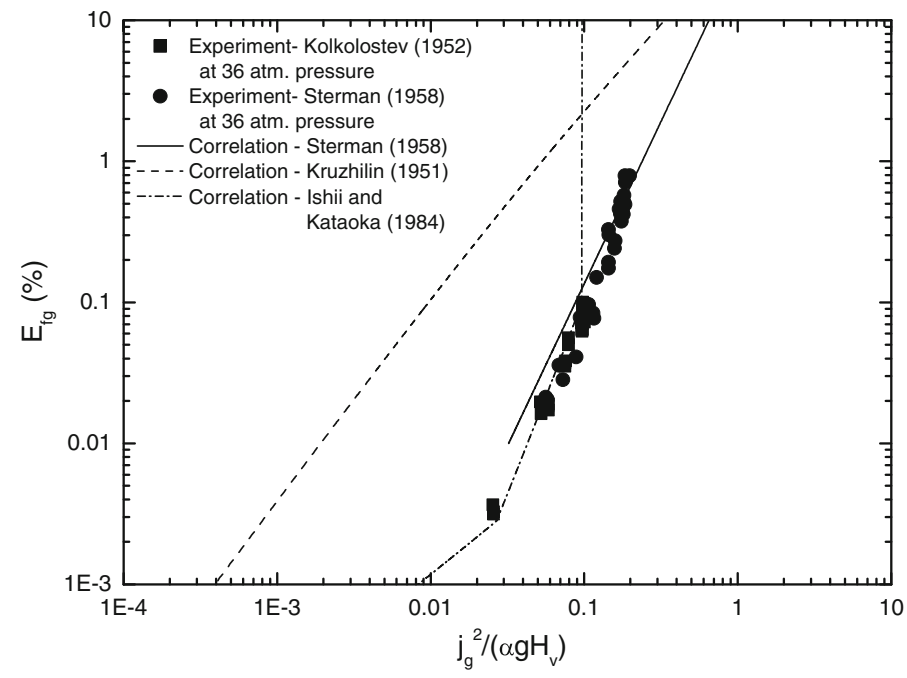

Figure 17. Prediction for Sterman (1958) and Kolkolostev (1952) at 36 atmospheric pressure. 


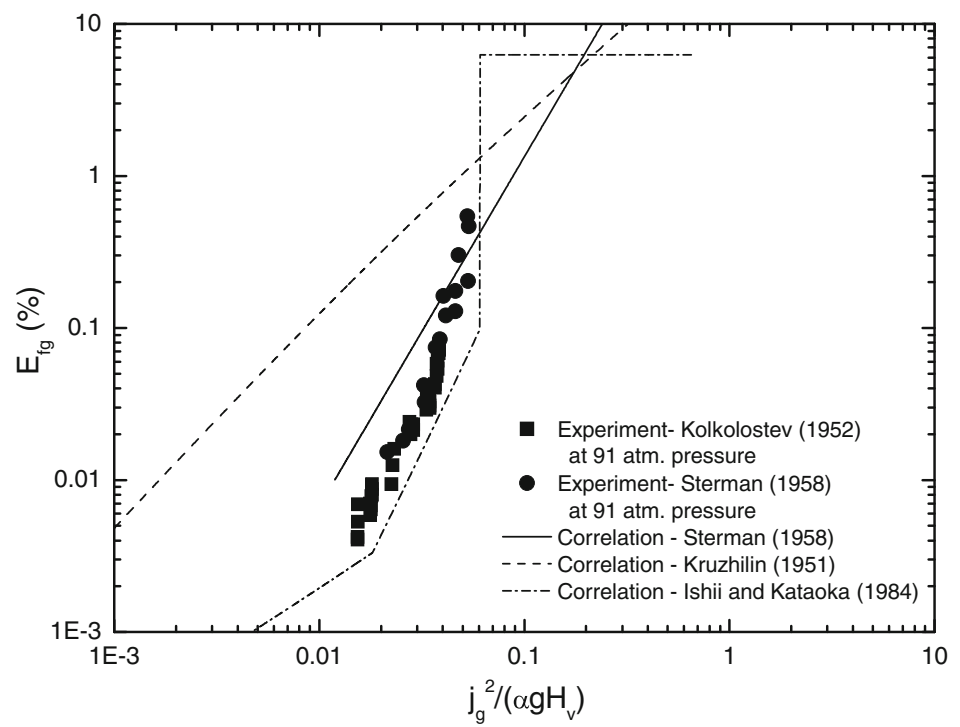

Figure 18. Prediction for Sterman (1958) and Kolkolostev (1952) at 91 atmospheric pressure.

Kataoka (1984). The entrainment factors predicted in upper momentum controlled region agreed well with the correlation but overall trend in the momentum controlled region and entrainment in deposition controlled region was measured to be as high as 5 times the prediction by Ishii \& Kataoka (1984) correlation. This difference was attributed to the following possible reasons.

a) The fit of Ishii \& Kataoka (1984) correlation is based on the data with higher superficial velocities $(>0.5 \mathrm{~m} / \mathrm{s})$ whereas the superficial velocities in the experiment were between 5 to $27 \mathrm{~mm} / \mathrm{s}$.

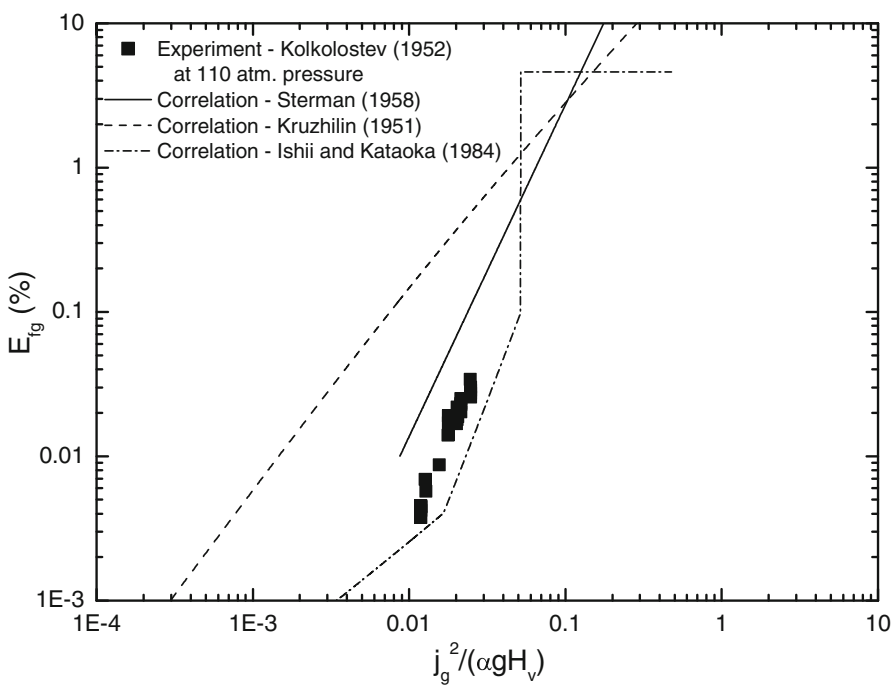

Figure 19. Prediction for Sterman (1958) and Kolkolostev (1952) at 110 atmospheric pressure. 
b) Concentration of solid tracer particles in and above the pool can affect the entrainment. This has not been accounted for in the Ishii \& Kataoka (1984) correlation. Therefore it can be inappropriate to compare the experimental results with the correlation by Ishii \& Kataoka (1984).

It was also stated that the Ishii \& Kataoka (1984) correlation was good for the prediction of solid fission products in air-steam atmosphere above the pool, but was erroneous for pure steam atmosphere as the effect of pressure is not properly described.

Iyer et al (2010) studied the effect of superficial gas velocity and the diameter ratio (of vessel to inlet pipe) on the carryover. The experiments were performed at atmospheric conditions with air and water mixture. The carryover was measured with the help of tissue papers suspended above the separation interface and the collected amount of entrainment was weighed. The entrainment was measured with vessel to inlet diameter ratio $=1$, where the bubbles were generated through grid plate with hole diameter $5 \mathrm{~mm}$. It was found to agree very well with the Ishii \& Kataoka (1984) correlation. With diameter ratio more than unity it was observed to be higher than the prediction by correlation and this was attributed to the jetting effect of two-phase mixture that issues from the riser. Thus in the first case with grid plates it appears that the bubbles at the interface had uniform distribution while without grid plate the separation interface is turbulent and there is splashing of liquid. Author concluded that thus the mechanism of droplet generation and associated size and velocity distribution considered by Ishii \& Kataoka (1984) may not hold good for the experimental case.

\section{Application of numerical methods and computational fluid dynamics}

\subsection{Preamble}

The investigations discussed in earlier sections, dealt with use of mathematical tools for the prediction of flow pattern. There are some additional tools on the basis of force and energy balances (Joshi \& Sharma 1976, 1979; Joshi \& Shah 1981; Joshi 1983; Pandit \& Joshi 1983, 1984, 1986; Joshi et al 1990). During the past 25 years computational fluid dynamics is being increasingly used because of the developments in the computational power as well as numerical techniques (Ranade \& co-workers 1989a, b, 1990a, b, 1992; Thakre \& Joshi 1999; Joshi et al 2002; Bhole et al 2007; Tabib et al 2008; Murthy \& Joshi 2008). In the published literature, the knowledge of flow pattern has been employed for the estimation of equipment performance such as mixing (Joshi \& Sharma 1978; Joshi 1980, 1982; Ranade et al 1991; Patwardhan \& Joshi 1999; Sahu et al 1999; Nere et al 2003; Kumaresan \& Joshi 2006), heat transfer (Joshi et al 1980; Dhotre \& Joshi 2004a), sparger design (Dhotre et al 2004b; Kulkarni et al 2009), gas induction (Joshi \& Sharma 1977; Murthy et al 2007a), solid suspension (Raghav Rao et al 1988; Rewatkar \& Joshi 1991; Murthy et al 2007b). Joshi \& Ranade (2003) have discussed the perspective of computational fluid dynamics (CFD) in designing process equipment with their views on expectations, current status and path forward.

\subsection{Simulation of vapour-liquid dispersion}

Vapour-liquid dispersion in the domain below the separation interface is governed by the equation of change for the two-phase flow. The dispersed vapour phase in the liquid exhibits various flow regime/patterns depending on the superficial velocities of the phases and hold-up profiles. 
The formulation of two-phase flow equations, underlying assumptions made for simplification, various force terms required for modelling the momentum exchange between the vapour and liquid phase, numerical modelling methodologies and relation of various parameters to the equipment design and related developments have been earlier critically reviewed by Joshi (2001).

\subsection{Simulation of drop-vapour dispersion}

The drop-vapour dispersion modelling is performed to analyze spray dryers, coal and liquid fuel combustion process and particle laden turbulent flows. Lagrangian description of droplets or particles is found to be most suitable for description of flows wherein the droplet or particle concentrations are low of the order of $10^{-2}$. The Eulerian approach is applicable for high concentration of dispersed phase. The numerical modelling of drop-vapour dispersion has been extensively reviewed by Jamaleddine \& Ray (2010), Langrish \& Fletcher (2001).

\subsection{Simulation of droplet formation at the interface between liquid phase and vapour phase}

The numerical simulations related to entrainment phenomenon in bubbling pools is mostly limited to the calculations upon the bubble bursting phenomenon and attempts to predict the generation of liquid droplets and their initial momentum. The subject area of droplet size distribution and velocity distribution at the bubbling interface is of utmost importance as basic assumptions on these distributions affect greatly the overall prediction of entrainment patterns above the separation interface. The droplet ballistic is relatively well developed subject area and thus can be efficiently dealt with known limitations.

The numerical simulation of basic process of droplet generation due to bursting bubbles is again limited to only analysis of the production of jet droplets. The process of droplet generation due to the thin film rupture is not studied in specifically, numerically as well as experimentally. Number of experimental investigations upon production of jet droplet is available in literature to compare with numerical predictions. The various numerical methodologies used in the past for prediction of jet droplet are presented in the following sections.

5.4a Boundary integral method (BIM): Boulton-Stone \& Blake (1993) carried out modelling of free surface motions following the burst of air bubble using BIM. In simulation it is assumed that when a rising bubble, with small terminal velocity arrives at the free surface, it reaches an equilibrium position before bursting. Figure 20 shows the schematic of a stable bubble at the interface. The equilibrium position of the bubble can be determined by microscopic balance applied on the three interfaces formed at bubble, film and meniscus denoted by $\Sigma_{b}, \Sigma_{f}$ and $\Sigma_{m}$ respectively. The principal radii of curvature for bubble, i.e., $R_{1}$ and $R_{2}$ are determined from the force balance at the point $\mathrm{C}$. The bubble film geometry is assumed to be described by part of sphere. By photographic evidences it is known that the Reynolds number for bubble during the bursting time is of the order of 1000 and vortices are limited to the boundary layers around thin layers of water/air interface, therefore the velocity distribution in the liquid domain can be represented by potential flow. Flow in the surrounding pool of liquid is considered to be inviscid. The motion of the liquid domain is described by the Laplace and Euler equations. These equations are solved in time domain. The local velocity potential at the bubble-liquid interface is applied as dynamic boundary condition. It is obtained by considering momentum balance at any point on interface. The detailed discussion for formulation and solution techniques is omitted here and details can be found in references. Boulton-Stone \& Blake (1993) performed calculations for jet 


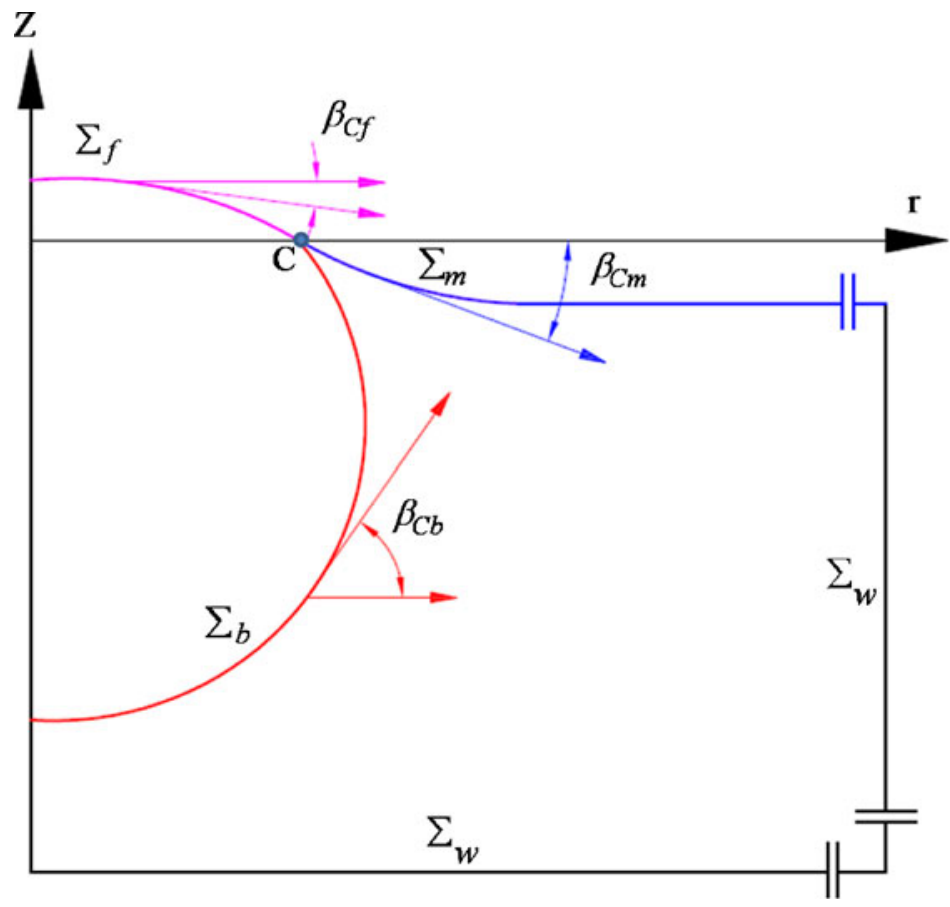

Figure 20. Gas bubble at a free liquid surface and resulting three joined surfaces (taken from Boulton Stone and Blake 1993).

formation and breakup followed by bubble burst for air-water environment at atmospheric pressure. The bubble diameters considered for the simulation were $0.05,0.075,0.1,0.2,0.25$ and $0.3 \mathrm{~cm}$. The simulations showed that only the small diameter bubbles can cause the ejection of droplet upon bursting (see figure 21). Upon increasing the bubble diameter the jet droplet is not formed due to insufficient potential available which is dictated by the geometry of the stabilized
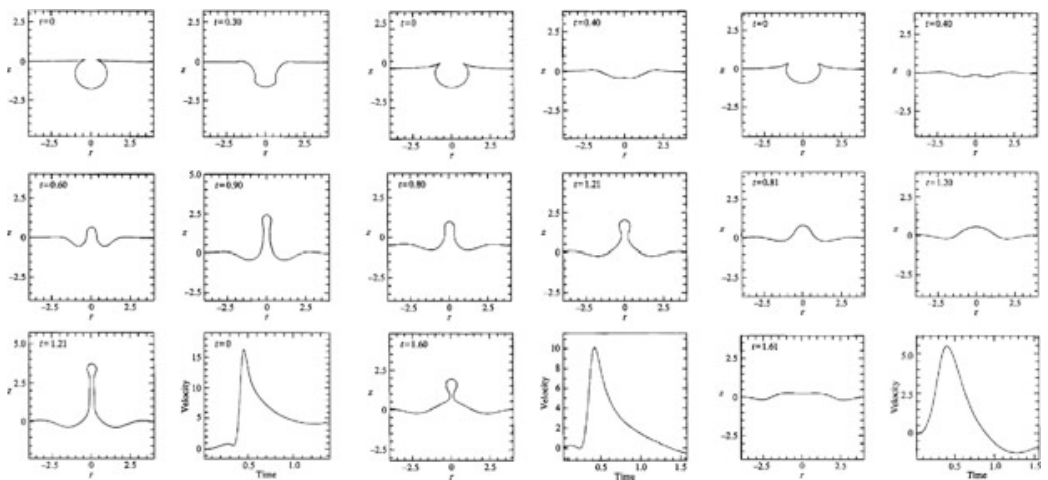

(a)

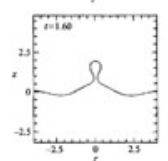

(b)

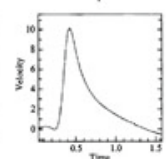

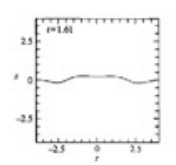

(c)

Figure 21. Calculated free surface shapes for bubbles of diameter (a) 0.75 , (b) 2.0 and (c) $3.0 \mathrm{~mm}$ diameter (taken from Boulton Stone and Blake 1993). 
bubble at the separation interface. This observation was in-line with the experiments by Keintzler et al (1954) and Garner et al (1954). Simulations showed that larger bubbles form wider jets upon bursting as observed in experiments. The surface shapes before jet production during collapse of $0.075 \mathrm{~cm}$ bubble were compared with Keintzler et al (1954) and it was found to have slight differences. The jet shape was asymmetric as per experiments and the time at which it disintegrates to form droplets was earlier than predicted. The entrainment rates were compared with Garner et al (1954) (see figure 22) and it was found to agree for smaller bubble diameter $(<1.5 \mathrm{~cm})$. For the larger bubble diameters the predicted jet drop volumes were significantly smaller than the experimental measurements. The critical bubble diameter above which no jet droplet forms was predicted to be $0.25 \mathrm{~cm}$ which was reasonably accurate with experimental value of $0.2 \mathrm{~cm}$.

Calculations with the method adopted could be only continued up to the formation of first droplet, which is major drawback as in reality there are multiple droplets formed due to jet disintegration. Also the relocation of nodes during jet disintegration to capture curved surfaces accurately was recommended to be improved upon in order to improve the simulations as differences with experimental observation were noted.

Georgescu et al (2002) carried out similar simulations as performed by Boulton-Stone \& Blake (1993). Surface evolution obtained for a case of $1.5 \mathrm{~mm}$ diameter bubble is shown in figure 23. Predictions of velocity of first jet droplet were comparable with the experiments. The comparison of dimensions of the first jet droplet is as shown in figure 24 below. Their prediction of critical bubble diameter is as shown in figure 25. Authors investigated the effect of water temperature in the range of $0-30^{\circ} \mathrm{C}$. They concluded that the geometry and velocity of first jet droplet depends more on the surface tension than the density or viscosity. The comparison of critical bubble diameter for viscid and inviscid case suggested that the viscosity tends to delay the formation of droplet and droplet diameter is slightly increased.

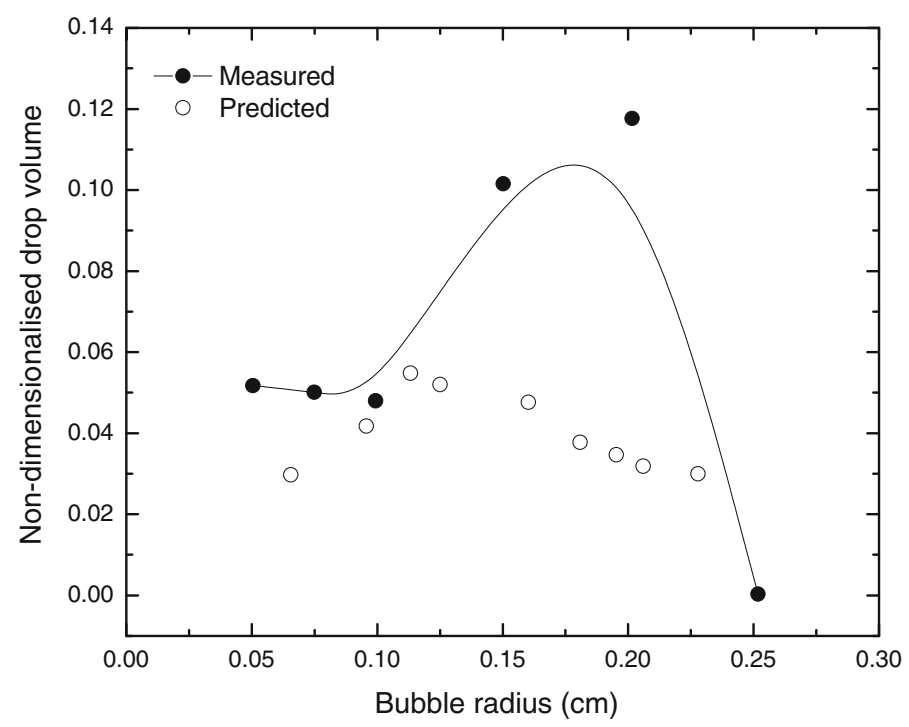

Figure 22. Non dimensional drop volume compared with Garner et al (1954) (taken from Boulton Stone and Blake 1993). 

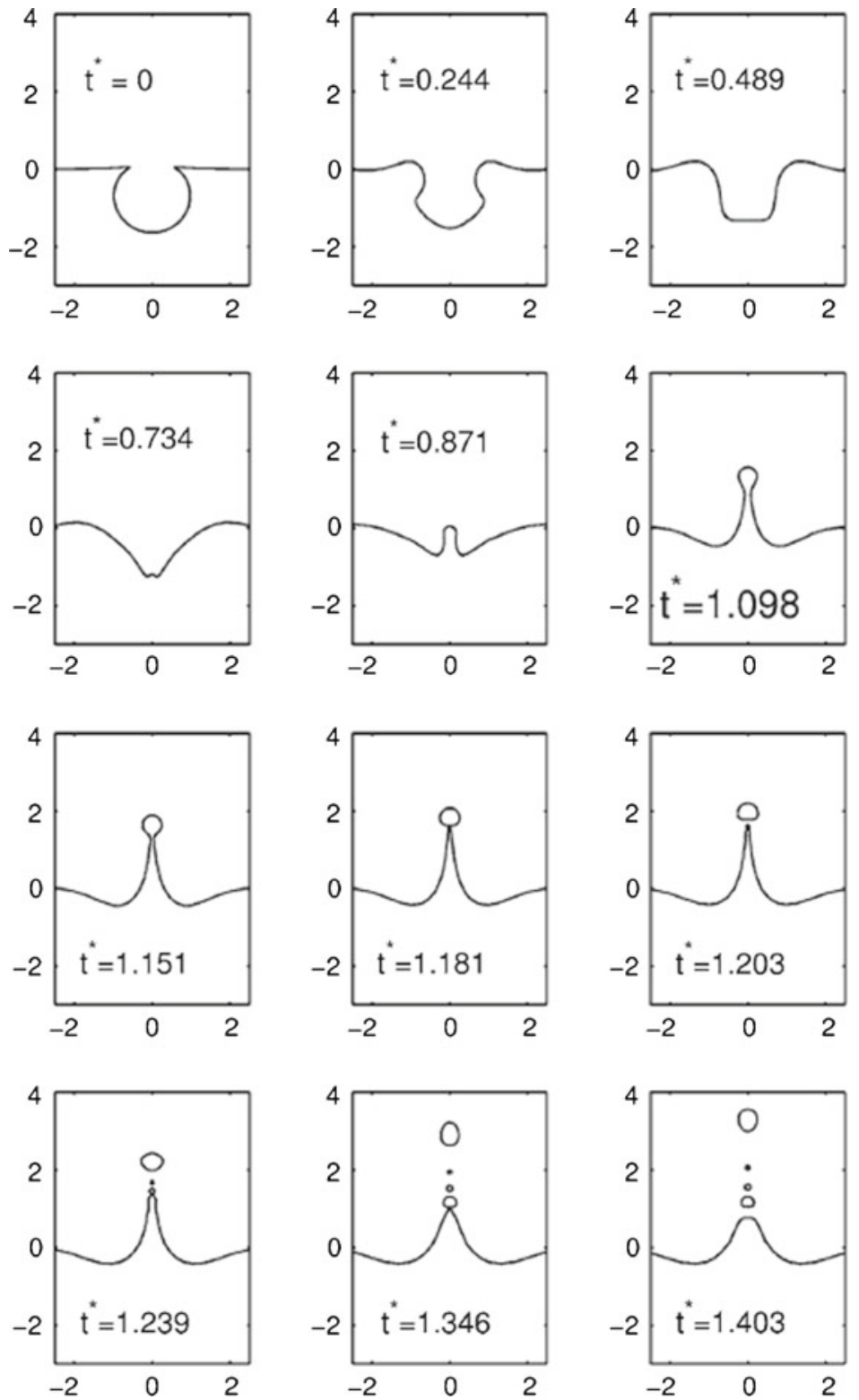

Figure 23. Surface evolutions for bubble of diameter $1.5 \mathrm{~mm}$ (taken from Georgescu et al 2002).

5.4b Level set method: Sussman \& Smereka (1997) carried out simulation of threedimensional axisymmetric free boundary for two immiscible fluids i.e., air and water. Motion of both the fluids is assumed to be governed by the incompressible Navier-Stokes equation. The interface between the fluids $\Gamma$ is the zero level set of $\phi$.

$$
\Gamma=\{x \mid \phi(x, t)=0\},
$$




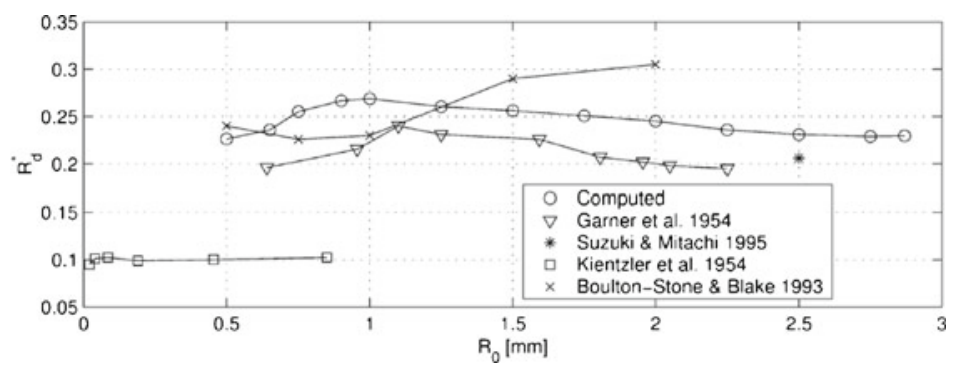

Figure 24. Jet drop dimensionless radius against the dimensional equivalent bubble radius $\mathrm{mm}$ (taken from Georgescu et al 2002).

also

$$
\phi(x, t)\left\{\begin{array}{ll}
>0 & x \in \text { liquid } \\
=0 & x \in \Gamma \\
<0 & x \in \text { gas }
\end{array} .\right.
$$

Thus the domain which is discontinuous at interface is now can be expressed as continuous function of level set $\phi$. Velocity is also continuous by defining as follows:

$$
V= \begin{cases}V_{l}, & \phi>0 \\ V_{g}, & \phi \leq 0\end{cases}
$$

The evolution of surface is then be given by

$$
\frac{\partial \phi}{\partial t}+V \cdot \nabla \phi=0
$$

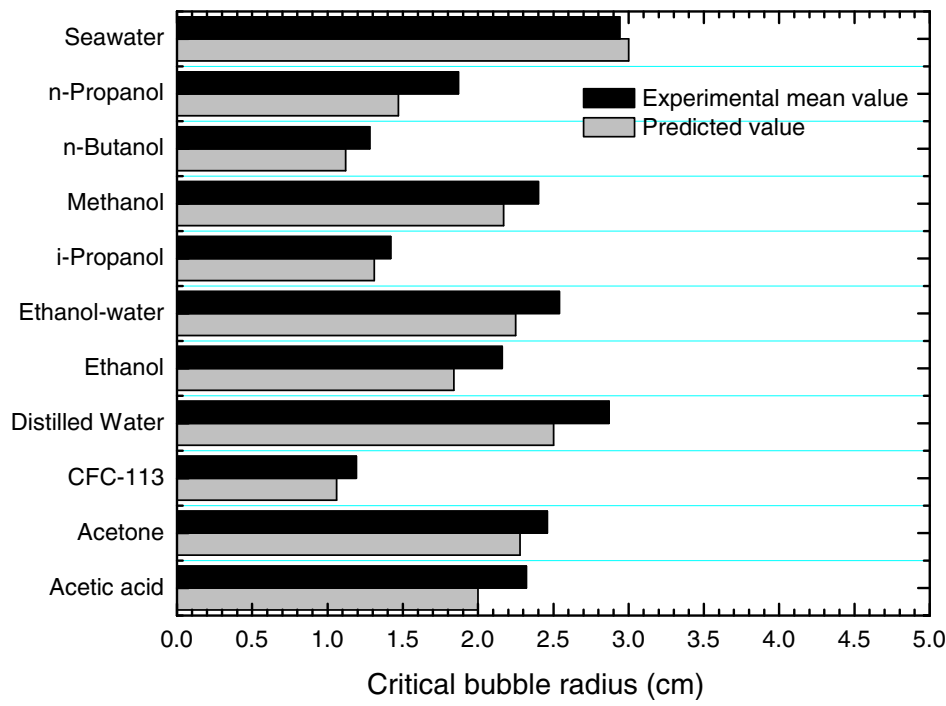

Figure 25. Comparison of prediction of critical bubble radius for various fluids $\mathrm{mm}$ (taken from Georgescu et al 2002). 
To determine the gas-liquid interface evolution, above equation is first parameterized with coordinates $(x, y)$. The resulting equation is then non-dimensionlized. The fluid properties like density and viscosity are also expressed as continuous function using Heaviside function. The velocity terms are represented by stream functions in the final equation of motion valid over entire domain. The detailed formulation and solution procedure can be found in the references. Sussman \& Smereka (1997) carried out numerical simulation of air bubble of size 4 and $5 \mathrm{~mm}$ diameter bursting at free surface (see figures 26 and 27). These bubbles were considered to be released just below the free surface. The computational resources limited, they could not simulate bubbles smaller than $4 \mathrm{~mm}$ size. The simulations are thus only qualitatively compared with earlier studies.

Though experimentally the critical bubble radius is $2 \mathrm{~mm}$, authors obtained jet droplet production for $4 \mathrm{~mm}$ bubble diameter considered in simulation. However, the advantage of level set method was demonstrated as the calculations could continue beyond the formation and detachment of first jet drop. Predicted jet velocity variation was matching qualitatively with that of given by Boulton-Stone \& Blake (1993).

Author studied the bursting phenomenon of bubble of the size ranging from 0.35 to $1.5 \mathrm{~mm}$ to predict the jet height and maximum velocity attained (figure 28). These predictions were in good agreement with the data from Spiel (1994).

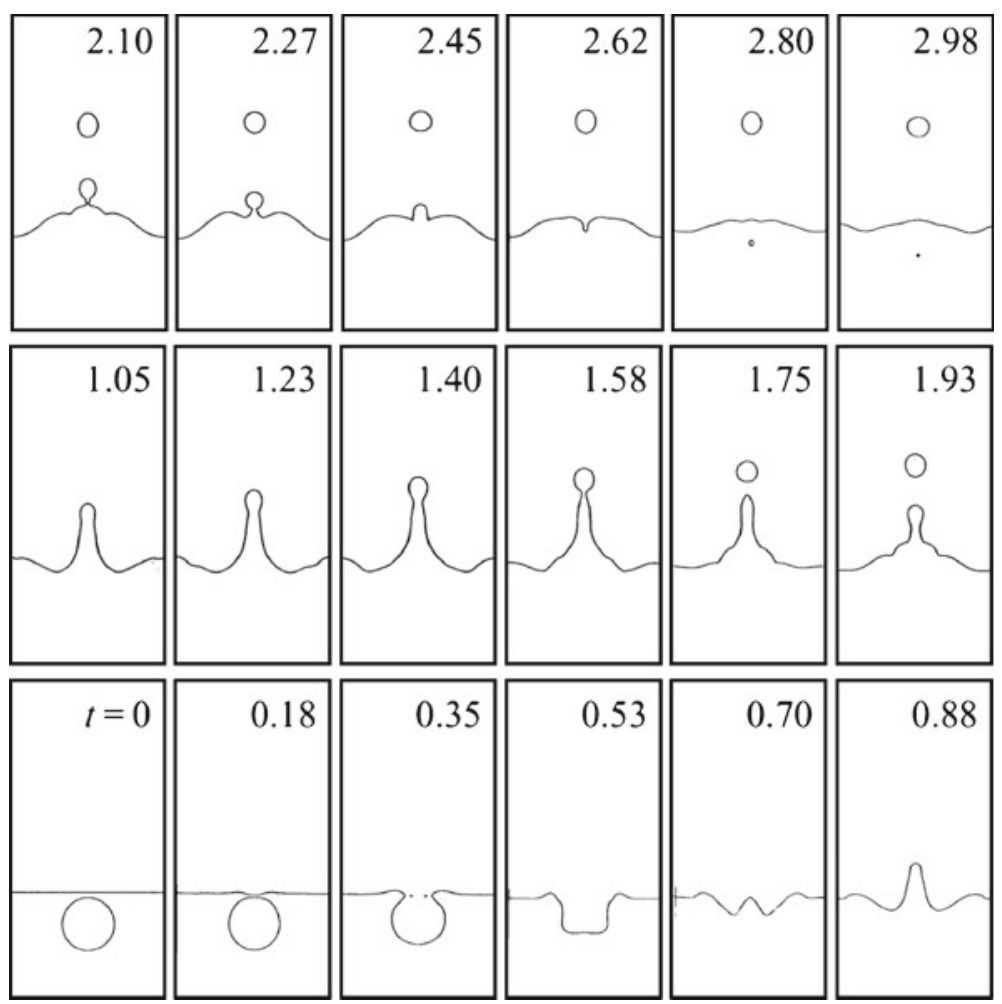

Figure 26. Evolution of a water jet resulting from a submerged gas bubble of $5 \mathrm{~mm}$ diameter (taken from Sussman \& Smereka 1997). 


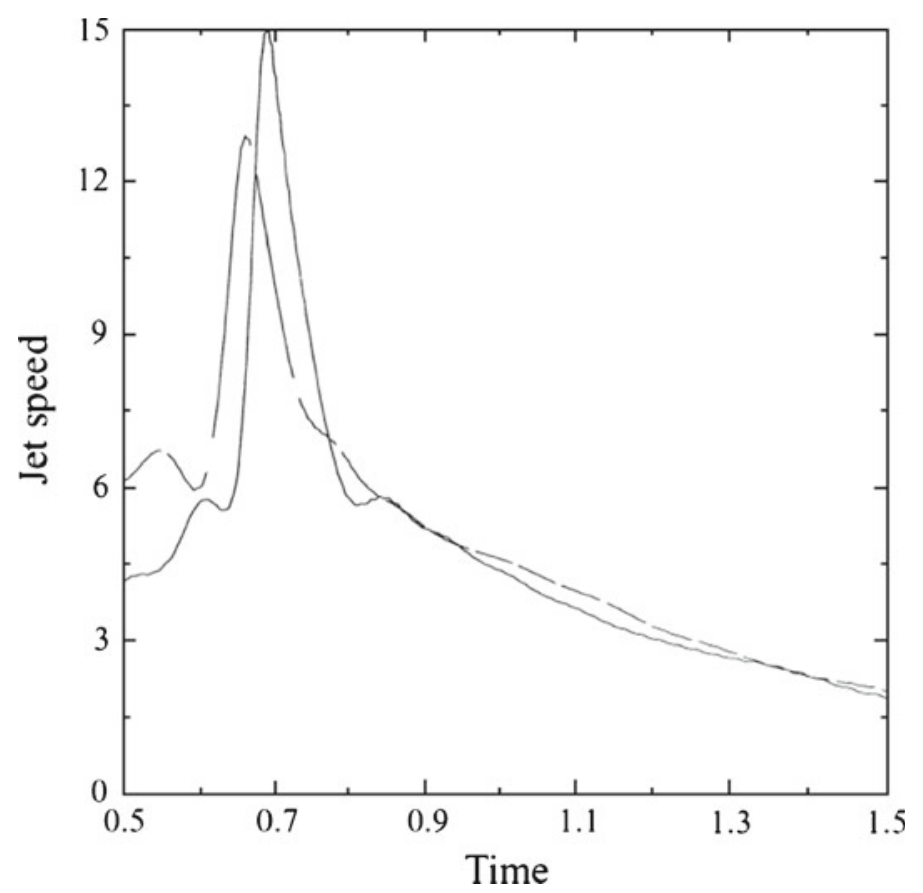

Figure 27. Jet velocity predicted for bubble of $4 \mathrm{~mm}$ (dotted line) and $5 \mathrm{~mm}$ (solid line) diameter (taken from Sussman \& Smereka 1997).

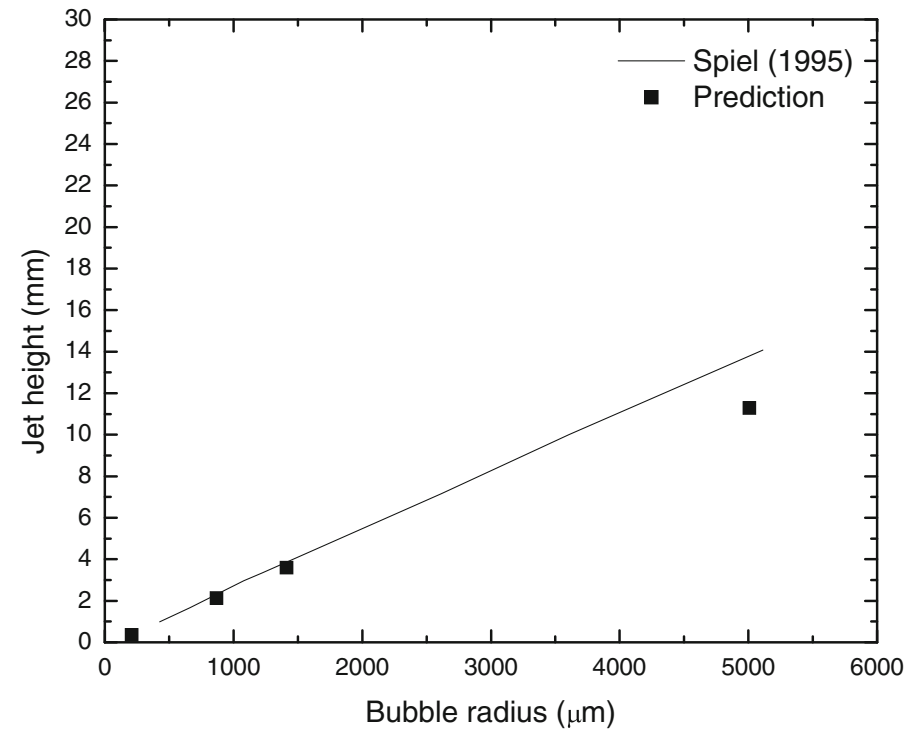

Figure 28. Comparison of predicted jet height with experimental data of Spiel (1994) (taken from Sussman \& Smereka 1997). 
5.4c Volume tracking method: Rudman (1998) carried out numerical simulation of bubble bursting through free surface with volume tracking method. In this method the equation of conservation of mass is described by

$$
\frac{\partial C}{\partial t}+\nabla \cdot(U C)=0
$$

where $C=$ the fractional volume function. The fluid density is described as:

$$
\rho=\rho_{1} C+\rho_{2}(1-C) .
$$

Navier-Stokes equation is assumed to be governing and momentum equation can be written as.

$$
\frac{\partial \rho U}{\partial t}+\nabla \cdot(\rho U U)=-\nabla p+\frac{1}{F r^{2}} \rho g+\frac{1}{W e} F_{S}+\frac{1}{\operatorname{Re}} \nabla \cdot \tau,
$$

where $F_{s}=$ surface force from interfacial forces.

$$
\mathrm{Fr}=\frac{\vartheta}{(g \ell)^{1 / 2}}, \quad \mathrm{We}=\frac{\rho \vartheta^{2} \ell}{\sigma}, \quad \mathrm{Re}=\frac{\rho \vartheta \ell}{\mu},
$$

where $\vartheta$ and $\ell$ are suitably chosen velocity and length scales respectively. The details of solution algorithm can be found in the reference.

The simulations by Rudman (1998) were carried out on 2-D grid. The density ratio of fluids was taken as 1000:1, and with constants $\mathrm{Fr}=1, \mathrm{We}=25$ and $\mathrm{Re}=500$. The initial circular bubble below the interface was observed to become cap-shaped bubble while approaching towards free surface. At interfaces it flattens and widens and thin film of liquid was observed to be formed. A thin film formed above the flat bubble then starts thinning and at the end droplets are ejected while rupture of the film takes place (figure 29). No comparison was made with experiments. The numerical simulations with VOF demonstrate the formation of film droplets, however only qualitatively so far.

5.4d Front tracking method: Duchemin et al (2001) studied the bubble bursting at free surface and subsequent jet formation using front tracking method. In this method, the incompressible Navier-Stokes equation is assumed to describe the motion of both fluids. The surface tension is considered to be a force concentrated at the interface. A characteristic function $\chi$ is defined such as, $\chi$ is 1 in one phase and 0 in another phase. The advection equation for $\chi$ is written as

$$
\frac{\partial \chi}{\partial t}+U \cdot \nabla \chi=0
$$

The density and viscosity are written as

$$
\begin{aligned}
& \rho=\chi \rho_{f}+(1-\chi) \rho_{g}, \\
& \mu=\chi \mu_{f}+(1-\chi) \mu_{g} .
\end{aligned}
$$

The equation of motion can be written in jump condition form. Jump conditions appear when we near the interface $S$. The solution of governing equation is solved on uniform Cartesian mesh $(\Delta x=\Delta y)$ using a staggered marker and cell distribution. The details of solution algorithm 


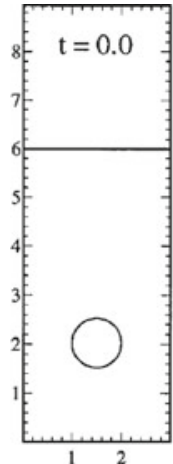

(a)

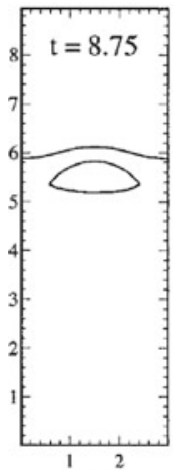

(f)

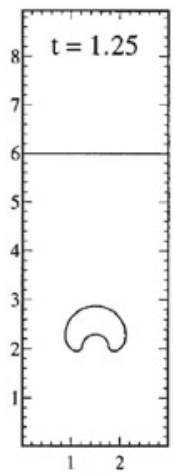

(b)

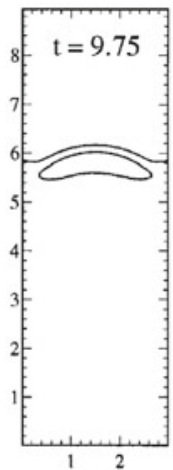

(g)

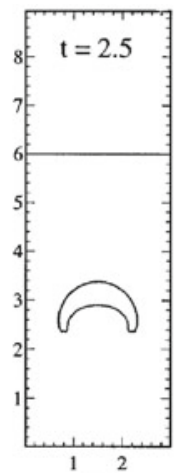

(c)

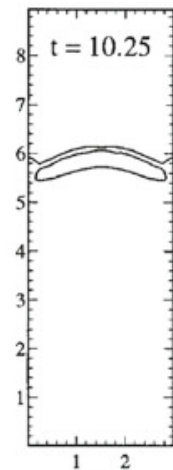

(h)

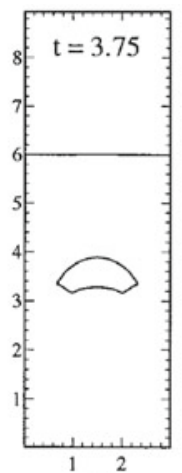

(d)

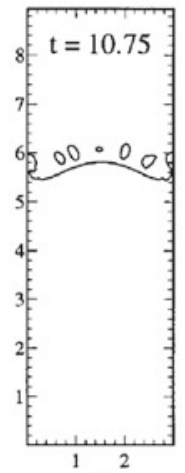

(i)

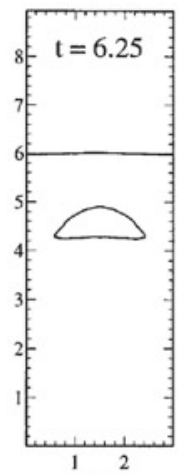

(e)

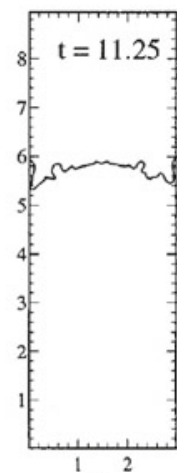

(i)

Figure 29. Rise of a 2D cylindrical bubble bursting through a free surface (taken from Rudman 1998).

can be found in the reference. Duchemin et al (2001) compared the surface evolutions with the surface shapes experimentally obtained by MacIntyre (1972).

Figure 30 illustrates the comparison of experimental and computational results. Authors conducted parametric study using computational method by varying the bubble size and predicted the jet velocity and size of the first jet drop formed after bubble burst. The jet droplet size prediction is shown in figure 31. Experiments by Spiel (1994) shows that the diameter of first jet drop

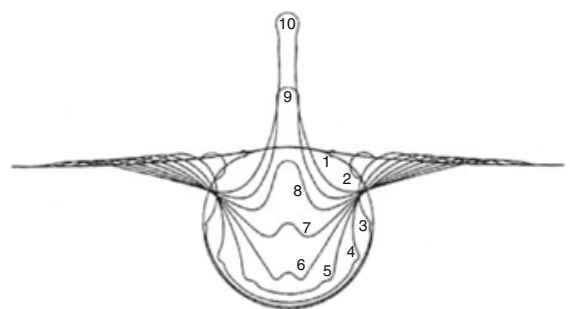

(a)

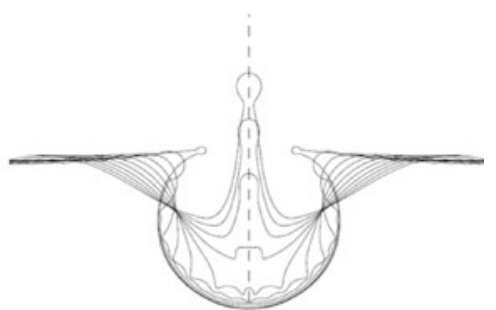

(b)

Figure 30. Time sequence of jet formation and bubble bursting at surface: (a) experimental profiles, (b) computational results. Numbers in figure (a) indicate different time instance (taken from Duchemin et al 2001). 


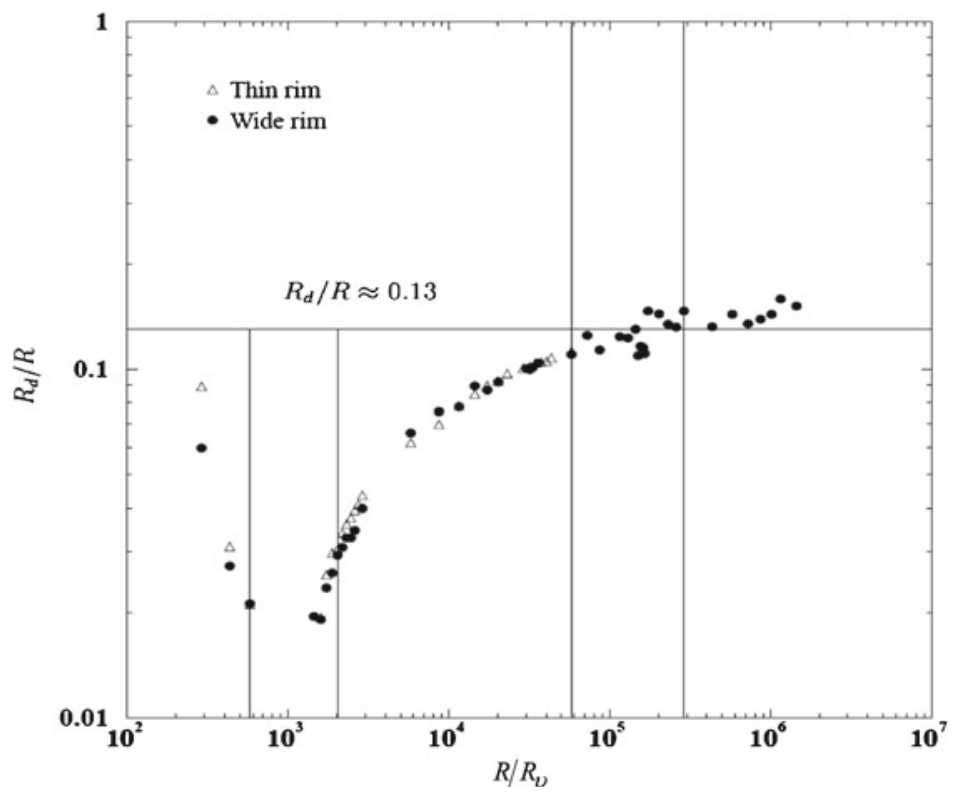

Figure 31. Ratio of radius of first ejected drop $R_{d}$ and bubble radius $R$ as a function of $R / R_{v}$ where $R_{v}=\rho v^{2} / \sigma$ (taken from Duchemin et al 2001).

formed is $10 \%$ of the bubble diameter. The numerical simulations by Duchemin et al (2001) shows that for large values of $R / R_{v}$ the predicted jet drop diameter was around $13 \%$ of the bubble diameter, which is consistent with findings of Spiel (1994). The predictions for small values $R / R_{v}$ were erroneous due to the jet size being thin and not resolved accurately by the numerical techniques employed.

All numerical methods described above deal with the formation of jet droplet, however there are other mechanisms of droplet formation at separation interface. The droplets formed due to rupture of thin liquid film of bubble dome have not been yet simulated by any numerical methods. The droplets formed due to shearing of liquid by the steam jetting through the separation interface are also not studied yet numerically. The level-set and front tracking methods as discussed above can be regarded as more suitable numerical techniques to determine the jet droplet formation. However, in presence of swarm of bubbles and turbulent interface these methods are not yet tested. This will be of practical interest to simulate exact bubble breakup process and formation of droplets at interface which act as a source term for carryover estimation.

\section{Conclusions}

In gravity separation of two-phase flows, the droplets generated at the interface depending on their size and initial momentum either fall back into liquid after travelling some distance inside the equipment or are carried along with the flow out of the equipment. This carryover can have adverse effects such as corrosion of piping and equipment due to presence of soluble salts. In case of power plant the carryover from steam generator can cause damage to steam turbine blades. In evaporators, the carryover reduces equipment efficiency and product yield. The complete elimination of carryover is however not possible. Additional equipments become necessary to 
reduce the carryover further. Therefore it is important to pay careful attention to the design of equipment to minimize the carryover by controlling the affecting parameters.

A review of the prediction procedures for the entrainment/carryover has been carried out. All the procedures consist of the following two steps:

(a) Estimation of liquid fragments, droplets present just above the separation interface.

(b) Prediction of the motion of such liquid droplets possessing some initial momentum and angle of ejection from separation interface in the bulk of the gas phase moving above the separation interface.

The above methodology is applicable where the separation of phases takes place purely by gravity and no mechanical equipments are employed to impart momentum on phases in favourable direction to control the settling of droplets.

Following are the important conclusions derived from the review of available literature and contributions of several researchers to the subject of entrainment/carryover in gas-liquid flows:

1. Experimental investigations on the basic input parameters to the process i.e., the entrainment flux at the interface in terms of droplet size and velocity distribution along with frequency of generation has been limited to the air-water systems or steam-water systems at low pressures. The following parameters are important:

a) Nature of turbulence at interface

b) Bubble dynamics below and at the interface

c) Governing mechanism of droplet formation.

The bubble dynamics and turbulence at the interface depend on the flow rates of the two phases, geometry of equipment and the fluid properties which in turn depend on operating parameters such as pressure and temperature. Owing to such multi-dimensional array of affecting parameters very few experiments are performed to study the role of these parameters. Also practical difficulties of instrumentation for visualization and measurement at high pressures have limited the scope of experiments. Therefore except few, the reliable data on these basic parameters do not exist.

2. A large number of simplifying assumptions have been made regarding the droplet size, velocity distribution by researchers investigating the subject analytically. These are either very conservative or sometimes irrelevant as the geometry and operating parameters of the concerned equipment differ from that of the equipment in which experiments were carried out to derive such distribution parameters. As far as the estimation of the liquid droplet dynamics in gas space is concerned the assumptions on the forces considered (drag, buoyancy and gravity) and their estimation appears to be conservative. Analytical studies have neglected the effect of flow pattern of bulk gas phase due to the complexity of gas flow pattern. This could present major limitation as the drag forces exhibit different behaviour when bulk is flow accelerated or decelerated.

3. Due to complexity of the subject, it is widely dealt with empirical approach and hence none of the attempts can be regarded as universally applicable. However, such correlations can provide quick estimates and augmentation with the experiments in equipment models can give better confidence in design. However, as per the investigations available in published literature, applicability of the empirical correlations has been seriously doubted because of unacceptable deviation from the experiment is found. This is mainly because; the two phase phenomenon has been incompletely understood together with possible cavitation (Pandit \& Joshi 1993) at the entry of steam-water mixture. 
4. Computational methods are promising in the view of availability of fast computing tools. Investigations have been very limited to the basic process of bubble bursting and droplet generation. However the CFD tools have great potential to model the basic two-phase processes taking place at the interface with techniques like Level set methods, VOF, Volume tracking and front tracking methods etc. The dispersed liquid phase in the gas space and gas bubbles in liquid can be efficiently modelled with population balance methods (Shah et al 1999). Though the CFD offers great potential to address the number issues in detail, a major task of validating these methodologies with relevant, reliable experimental data remains a major challenge.

5. The formation of the drops at the interface depends upon the quality of turbulence at the interface. The turbulence also governs the droplet dispersion in the gas phase. Therefore, it is important to identify the turbulence structures and understand their dynamics (Kulkarni et al 2001; Mathpati \& Joshi 2007; Joshi et al 2009; Mathpati et al 2009).

\section{Nomenclature}

$\begin{array}{ll}A & \text { Surface area of droplet } \\ A_{d} & \text { Projected area of droplet } \\ c_{1} & \text { Constant in Equation (19) } \\ C & \text { Fractional volume function } \\ C_{D} & \text { Drag coefficient of droplet } \\ D & \text { Droplet diameter } \\ D_{c} & \text { Critical droplet diameter } \\ D^{*} & \text { Dimensionless droplet diameter, } D / \sqrt{(\sigma / g \Delta \rho)} \\ D_{\text {max }}^{*} & \text { Dimensionless maximum droplet diameter } \\ D_{B} & \text { Bubble diameter } \\ D_{H} & \text { Hydraulic diameter of vessel } \\ D_{H}^{*} & \text { Dimensionless hydraulic diameter of vessel, } D_{H} / \sqrt{(\sigma / g \Delta \rho)} \\ D_{i} & \text { Diameter of inlet pipe to vessel } \\ D_{o} & \text { Diameter of outlet pipe from vessel } \\ D_{j} & \text { Diameter of jet } \\ E_{u} & \text { Euler number } \\ E_{f g} & \text { Entrainment fraction } \\ E_{f g}\left(h, J_{g}\right) & \text { Entrainment at height } h \text { and gas velocity } J_{g} \\ E_{0}\left(D, J_{g}\right) & \text { Entrainment at separation interface which consists of droplets whose diameter } \\ E_{f g}\left(D, J_{g}\right) & \text { is less than } D \\ & \text { Entrainment at any cross-section above the interface which consists of droplets } \\ f_{i} & \text { whose diameter is less than } D \\ f\left(D, J_{g}\right) & \text { Interfacial friction factor between gas and liquid } \\ \text { Fr } & \text { Droplet size distribution function for } J_{g} \\ F_{D} & \text { Froude number } \\ F_{s} & \text { Drag force on droplet } \\ G_{f} & \text { Surface force from interfacial forces } \\ g & \text { Entrainment flux } \\ g\left(V_{i}, D, J_{g}\right) & \text { Acceleration due to gravity } \\ H & \text { Height of the equipment } \\ & \end{array}$


$H_{V} \quad$ Height of vapour space inside the equipment

$H_{L} \quad$ Initial height of liquid in the equipment

$H_{M} \quad$ Height at which measurement is taken

$h \quad$ Height above the pool surface

$h_{j} \quad$ Height of rising jet

$h^{*} \quad$ Dimensionless height above the pool surface, $h / \sqrt{(\sigma / g \Delta \rho)}$

$h_{m} \quad$ Maximum height attained by rising droplet

$h_{f g} \quad$ Latent heat of evaporation

$J_{g} \quad$ Superficial velocity of gas

$J_{f} \quad$ Superficial velocity of liquid

$J_{g}^{*}$

$k$

$K \quad$ Drag force constant, $R /\left(V_{d}-V_{g}\right)^{2}$

$l \quad$ Length of liquid ligament

$m_{e f} \quad$ Density of massive flow of liquid in $\mathrm{kg} \cdot \mathrm{sec}^{2} / \mathrm{m}$

$m_{e g} \quad$ Density of massive flow of gas in $\mathrm{kg} \cdot \mathrm{sec}^{2} / \mathrm{m}$

$n \quad$ Normal to the interface

$N_{\mu g} \quad$ Gas viscosity number, $\mu_{g} /\left(\rho_{g} \sigma \sqrt{\sigma /(g \Delta P)}\right)^{1 / 2}$

$N_{\mu f} \quad$ Liquid viscosity number, $\mu_{f} /\left(\rho_{f} \sigma \sqrt{\sigma /(g \Delta P)}\right)^{1 / 2}$

$P \quad$ System pressure

$P_{i} \quad$ Pressure at the $i$

$P_{1} \quad$ Pressure inside bubble

$P_{o} \quad$ Pressure around the bubble

$q \quad$ Terminal velocity of droplet, $\sqrt{W / K}$

$r \quad$ Radial coordinate

$R \quad$ Resistance due to drag

$R_{f} \quad$ Radius of film

$R_{1}, R_{2} \quad$ Principal radii of bubble

$R_{1}^{*}, R_{2}^{*} \quad$ Non dimensionalized principal radii of bubble

$R_{0} \quad$ Equivalent bubble radius

$R_{d} \quad$ Radius of droplet

$R_{b} \quad$ Radius of bubble

$R_{v} \quad$ Length scale for bubble, $R_{v}=\rho v^{2} / \sigma$

$\operatorname{Re}_{D} \quad$ Droplet Reynolds number, $\rho_{g} V_{f} D / \mu_{g}$

$\operatorname{Re}_{g} \quad$ Gas Reynolds number, $\rho_{g} J_{g} D_{H} / \mu_{g}$

$s \quad$ Total surface area of droplets

$t \quad$ Time

$t^{*} \quad$ Non dimensionalized time

$t_{b} \quad$ Bubble burst time

$T_{s} \quad$ Saturation temperature

$\Delta T \quad$ Overcooling of droplet compared with the saturation temperature

$U, V \quad$ velocity

$u_{x} \quad x$-component of velocity

$V^{*} \quad$ Non dimensionalized velocity

$V_{d} \quad$ Droplet velocity

$V_{f} \quad$ Velocity of liquid filament

$V_{g} \quad$ Gas velocity 


$\begin{array}{ll}V_{g}^{*} & \text { Dimensionless gas velocity, } V_{g} / \sqrt{\left(\sigma g \Delta \rho / \rho_{g}^{2}\right)^{1 / 4}} \\ V_{h}\left(D, J_{g}, h\right) & \text { Initial velocity of droplet (diameter } D) \text { necessary to rise more than height } h \\ & \text { under gas velocity } J_{g} \\ V_{h}^{*} & \text { Dimensionless form of } V_{h}, V_{g} / \sqrt{\left(\sigma g \Delta \rho / \rho_{g}^{2}\right)^{1 / 4}} \\ V_{i} & \text { Initial velocity of droplet at pool surface } \\ V_{i}^{*} & \text { Dimensionless initial velocity, } V_{i} / \sqrt{\left(\sigma g \Delta \rho / \rho_{g}^{2}\right)^{1 / 4}} \\ V_{r} & \text { Relative velocity of droplet, } V_{d}-V_{g} \\ W & \text { Weight of droplet } \\ \text { We } & \text { Weber number } \\ w & \text { Dryness fraction of steam } \\ x & \text { Coordinate in horizontal direction } \\ y & \text { Vertical position above pool surface } \\ z & \text { Vertical coordinate }\end{array}$

\section{Greek symbols}

$\begin{array}{ll}\alpha & \text { Void fraction of the liquid pool } \\ \beta & \text { Angle in radian } \\ \phi & \text { Level set function } \\ \phi_{p} & \text { Velocity potential } \\ \phi_{p}^{*} & \text { Non dimensionalized potential function } \\ \psi & \text { Stream function } \\ \Delta \rho & \text { Density difference between gas and liquid } \\ \Delta P & \text { Pressure difference between inside and outside of drop } \\ \dot{\varepsilon}\left(J_{g}\right) & \text { Entrainment rate at the pool surface for } J_{g}\left[\mathrm{~kg} \mathrm{~m}^{-2} \mathrm{~s}^{-1}\right] \\ \mu_{f} & \text { Viscosity of liquid } \\ \mu_{g} & \text { Viscosity of gas } \\ \rho_{f} & \text { Density of liquid } \\ \rho_{g} & \text { Density of gas } \\ v & \text { Kinematic viscosity of fluid } \\ \sigma & \text { Surface tension } \\ \tau_{i} & \text { Interfacial shear stress between gas and liquid ligament } \\ \Gamma & \text { Gas-liquid interface } \\ \delta & \text { Delta-Dirac function } \\ \vartheta & \text { Velocity scale } \\ \ell & \text { Length scale } \\ \Delta x & \text { Cartesian mesh interval length in } x \text {-direction } \\ \Delta y & \text { Cartesian mesh interval length in } y \text {-direction } \\ \zeta & \text { Deformation tensor } \\ \Pi_{1} & \text { Dimensionless number in Equation (13) } \\ \Pi_{2} & \text { Dimensionless number in Equation (14) } \\ \Pi_{3} & \text { Dimensionless number in Equation (15) } \\ \Pi_{4} & \text { Dimensionless number in Equation (16) } \\ \Sigma_{b} & \text { Interface formed at bubble } \\ \Sigma_{f} & \text { Interface formed at film } \\ \Sigma_{m} & \text { Interface formed at meniscus } \\ & \end{array}$


$\Sigma_{w} \quad$ Interface at wall

$\beta_{C f} \quad$ contact angle of film

$\beta_{C b} \quad$ contact angle of the bubble and liquid interface

$\beta_{C m} \quad$ contact angle of meniscus

\section{References}

Aiba S and Yamada T 1959 Studies on entrainment. A.I.Ch.E. J. 5(4): 506

Bhole M R, Joshi J B and Ramkrishna D 2007 Population balance modeling for bubble columns operating at the homogeneous reginme. A.I.Ch.E. J. 53: 579-588

Boulton-Stone J M and Blake J R 1993 Gas bubbles bursting at a free surface. J. Fluid Mech. 254: 4737

Cheng S I and Teller A J 1961 Free entrainment behaviour in sieve trays. A.I.Ch.E. J. 7(2): 283

Cosandy J O, Gunther A and von Rohr P R 2001 Entrainment of soluble and non soluble tracers from a boiling water surface. Nucl. Engg. Desgn. 208: 87

Cosandy J O, Gunther A and von Rohr P R 2003 Transport of salts and micron-sized particles entrained from a boiling water pool. Expt. Thermal and Fluid Science 27: 877

Davis R F 1940 The Physical Aspect of Steam Generation at High Pressures and Problem of Steam Contamination. Proc. Inst. Mech. Engrs. 144: 198-215

Dhotre M T and Joshi J B 2004a Two dimensional CFD model for flow pattern, heat transfer and pressure drop in bubble column reactor. Trans. Instn. Chem. Engrs: Chem. Eng. Res. Des. 82 A6: 689-707

Dhotre M T, Ekambara K and Joshi J B 2004b CFD simulation of sparger design and height to diameter ratio on gas hold-up profiles in bubble column reactors. Exper. Therm \& Fluid Sci. 28: 407-421

Duchemin L, Popinet S, Jpsserand C and Zaleski S 2001 Jet formation in bubbles bursting at a free surface. Phys. Fluids. 14: 3000-3008

Garner F H, Ellis S R M and Lacey J A 1954 The size distribution and entrainment of droplets. Trans. Instn. Chem. Engrs. 32: 222

Georgescu S C, Achard J L and Canot E 2002 Jet drops ejection in bursting gas bubble processes. European J. Mech. Fluids 21: 265

Gunther A, Walchli S and von Rohr P R 2003 Droplet production from disintegrating bubbles at water surfaces. Single vs. multiple bubbles. Int. J. Multiphase Flow 29: 795

Ishii M 1977 One-dimensional drift-flux model and constitutive equations for relative motion between phases in various flow regimes. ANL-77-47 Argone National Laboratory, Argone

Ishii M and Kataoka I 1984 Mechanistic modeling of pool entrainment phenomenon. Int. J. Heat and Mass Transfer 27(11): 1999

Iyer K, Prabhudharwadkar M and More R Z 2010 Experimental study of liquid carryover in a separator drum. Nucl. Engg. Desgn. 240: 76

Jamaleddine T J and Ray M B 2010 Application of computational fluid dynamics for simulation of drying processes: A review. Drying technology 28: 120-154

Joshi J B 1980 Axial mixing in multiphase contactors-a unified correlation. Trans. Instn. Chem. Engrs. 58: $155-165$

Joshi J B 1982 Gas phase dispersion in bubble columns. Chem. Eng. J. 24: 213-216

Joshi J B 1983 Solid-liquid fluidised beds-some design aspects. Trans. Instn. Chem. Engrs. 61: $143-161$

Joshi J B 2001 Computational flow modelling and design of bubble column reactors. Chem. Eng. Sci. 56: $5893-5933$

Joshi J B and Ranade V V 2003 Computational fluid dynamics for designing process equipment: expectations, current status and path forward. Ind. Eng. Chem. Res. 42: 1115-1128

Joshi J B, Ranade V V, Gharat V V and Lele S S 1990 Sparged loop reactors. Can. J. Chem. Eng. 68: $705-741$ 
Joshi J B and Shah Y T 1981 Hydrodynamics and mixing models for bubble column reactors. Chem. Eng. Commun. 11: 165-199

Joshi J B and Sharma M M 1976 Mass transfer characteristics of horizontal sparged contactors. Trans. Instn. Chem. Engrs. 54: 42-53

Joshi J B and Sharma M M 1977 Mass transfer and hydrodynamic characteristics of gas inducing type of agitated contactors. Can. J. Chem. Eng. 55: 683-695

Joshi J B and Sharma M M 1978 Liquid phase back-mixing in sparged contactors. Can. J. Chem. Eng. 56: $116-119$

Joshi J B and Sharma M M 1979 A circulation cell model for bubble columns. Trans. Instn. Chem. Engrs. 57: 244-251

Joshi J B, Vitankar V S, Kulkarni A A, Dhotre M T and Ekambara K 2002 Coherent flow structures in bubble column reactors. Chem. Eng. Sci. 57: 3157-3183

Joshi J B, Tabib M V, Deshpande S S and Mathpati C S 2009 Dynamics of flow structures and transport phenomena-I: experimental and numerical techniques for identification and energy content of flow structures. Ind. Eng. Chem. Res. 48: 8244-8284

Joshi J B, Sharma M M, Shah Y T, Singh C P P, Ally M and Klinzing G E 1980 Heat transfer in multiphase contactors. Chem. Eng. Commun. 6: 257-271

Keintzler C F, Arons A B, Blanchard D C and Woodcock A H 1954 Photographic investigation of the projection of the droplets by bubbles bursting at a water surface. Tellus 6(1): 1-7

Kim H and No H C 2005 Liquid entrainment and off-take through the break at the top of a vessel. Nucl. Engg. Desgn. 235: 1675

Kocamustafagullari G, Smits S R, Razi J and Huang W D 1993 Droplet size modeling in annular flow, Proc. Sixth International Tropical Meeting on Nuclear Reactor Thermal Hydraulics, NURETH-6, Vol. 2, 1021-1030

Koch M K, Voßnacke A, Starflinger J, Schutz W and Unger H 2000 Radionuclide re-entrainment at bubbling water pool surfaces. J. Aerosol Sci. 31: 1015

Kolkolostev V A 1952 Investigation of the operation of the steam space of the ISV evaporator, Trudy, MEI.

Kruzhilin G N 1951 The dependence of the permissible vapour load upon the pressure. Izv. Akad. Nauk. Otd. Tekh. Nauk. 7: 1106

Kulkarni A A, Joshi J B, Kumar V R and Kulkarni B D 2001 Application of multiresolution analysis for simultaneous measurement of gas and liquid velocities and fractional gas hold-up in bubble column using LDA. Chem. Eng. Sci. 56: 5037-5048

Kulkarni A V, Badgandi S V and Joshi J B 2009 Design of ring and spider type spargers for bubble column reactor: experimental measurements and CFD simulation of flow and weeping. Chem. Eng. Res. Des. 87(12): 1612-1630

Kumaresan T and Joshi J B 2006 Effect of impeller design on the flow pattern and mixing in stirred tanks. Chem. Eng. J. 115: 173-193

Langrish T A G and Fletcher D F 2001 Spray drying of food ingredients and applications of CFD in spray drying. Chem. Engg. and processing 40: 345-354

Mathpati C S and Joshi J B 2007 Insight into theories of heat and mass transfer at the solid-fluid interface using direct numerical simulation and large eddy simulation. Ind. Eng. Chem. Res. 46: 8825-8557

Mathpati C S, Tabib M V, Deshpande S S and Joshi J B 2009 Dynamics of turbulent structures and transport phenomena-2: relationship with design objectives and design optimization. Ind. Eng. Chem. Res. 46: $8285-8311$

MacIntyre F 1972 Flow patterns in breaking bubbles. J. Geophys. Res. 77(27): 5211-5228

Minges J, Schutz W and Koch M K 1998 KAREX-Experimentec zum radiologischen Quellterm infolge Resuspension, Projekt Sicherheitsforschung, Jahresbericht 1997, FZKA 6126, Forschungszentrum Karlsruhe, pp. 517-523

Mochizuki H and Hirao Y 1993 Development of steam separator performance analysis code and its validation-carryover experiments. J. Nucl. Sci. Tech. 30(10): 1059

Murthy B N and Joshi J B 2008 Assessment of standard k- $\varepsilon$, rsm and les turbulence models in a baffled stirred vessel agitated by various impeller designs. Chem. Eng. Sci. 63: 5468-5495 
Murthy B N, Deshmukh N, Patwardhan A W and Joshi J B 2007a Hollow self inducing impeller: flow visualisation and CFD simulation. Chem. Eng. Sci. 62: 3839-3848

Murthy B N, Ghadge R S and Joshi J B 2007b CFD simulations of gas-liquid-solid stirred reactor: prediction of critical impeller speed for solid suspension. Chem. Eng. Sci. 62: 7184-7195

Nayak A K, Jain V, Vijayan P K, Saha D and Sinha R K 2000 Study on the carryover behaviour in the steam drum of the advanced heavy water reactor, Sixteenth National Convention of Mechanical Engineers and All India Seminar on Future Trends in Mechanical Engineering, Sept 29-30, 2000

Nere N K, Patwardhan A W and Joshi J B 2003 Liquid phase mixing in stirred vessels: turbulent flow regime. Ind. Eng. Chem. Res. 42: 2661-2698

Newitt D M, Dombrowski N and Knelman F H 1954 Liquid entrainment - 1. The mechanism of drop formation from gas or vapour bubbles. Trans. Instn Chem. Engrs. 32: 244

Panasenko M D 1959 Correlation of mechanical carryover by steam. Teploenergetika, 1959

Pandit A B and Joshi J B 1983 Mixing in mechanically agitated contactors, bubble columns and modified bubble columns. Chem. Eng. Sci. 38: 1189-1215

Pandit A B and Joshi J B 1984 Three phase sparged reactors: some design aspects. Rev. Chem. Eng. 2: 1-84

Pandit A B and Joshi J B 1993 Hydrolysis of fatty oils - effect of cavitation. Chem. Eng. Sci. 48: 3440-3442

Pandit A B and Joshi J B 1986 Mass and heat transfer characteristics of three phase sparged reactors. Trans. Instn. Chem. Engrs. 64: 125-157

Patwardhan A W and Joshi J B 1999 Relation between flow pattern and blending in stirred tanks. Ind. Eng. Chem. Res. 38: 3131-3143

Raghav Rao K S M S, Rewatkar V B and Joshi J B 1988 Critical impeller speed for solid suspension in mechanically agitated solid liquid contactors. A.I.Ch.E. J. 34: 1332-1340

Ranade V V and Joshi J B 1989a Flow generated by pitched bladed turbine part I: experimental. Chem. Eng. Commun. 81: 197-224

Ranade V V, Joshi J B and Marathe A G 1989b Flow generated by pitched bladed turbine part II: mathematical modelling and comparison with experimental data. Chem. Eng. Commun. 81: 225-248

Ranade V V and Joshi J B 1990a Flow generated by a disc turbine I: experimental. Trans. Instn. Chem. Engrs. Chem. Eng. Res. Des. 68: 19-33

Ranade V V and Joshi J B 1990b Flow generated by a disc turbine II: mathematical model. Trans. Intsn. Chem. Engrs. Chem. Eng. Res. Des. 68: 34-50

Ranade V V, Bourne J R and Joshi J B 1991 Fluid mechanics and blending in agitated tanks. Chem. Eng. Sci. 46: 1883-1893

Ranade V V, Mishra V P, Saraph V S, Deshpande G B and Joshi J B 1992 Comparison of axial flow impellers using LDA. Ind. Eng. Chem. Res. 31: 2370-2379.

Rewatkar V B and Joshi J B 1991 Critical impeller speed for solid suspension in mechanically agitated three phase reactors I: experimental Part. Ind. Eng. Chem. Res. 30: 1770-1784

Rozen A M, Golub S I and Davydov I F 1970 Some laws governing drop carryover. Sovt. Phys. Doklady 14(7): 648

Rudman M 1998 A Volume-tracking method for incompressible multifluid flows with large density variations. Int. J. Numer. Meth. Fluids 28: 357-378

Sahu A K, Kumar P, Patwardhan A W and Joshi J B 1999 CFD modeling and mixing in stirred tanks. Chem. Eng. Sci. 54: 2285-2293

Shah Y T, Pandit A B and Mohalkar 1999 Cavitation Reaction Engineering. Limited edition, London, Springer

Spiel D E 1994 The sizes of the jet drops produced by air bubbles bursting on sea and fresh water surfaces. Tellus 46B: 325

Sterman L S 1958 On the theory of steam separation. Sovt. Phys. - Tech. Phys. 28(7): 1562

Sussman M and Smereka P 1997 Axisymmetric free boundary problems. J. Fluid Mech. 341: 269-294

Tabib M V, Roy S V and Joshi J B 2008 CFD simulation of bubble column: an analysis of interphase force and turbulence models. Chem. Eng. J. 139: 589-614

Thakre S S and Joshi J B 1999 CFD simulation of flow in bubble column reactors importance of drag force formulation. Chem. Eng. Sci. 54: 5055-5060 
Voßnacke A, Koch M K and Unger H 1997 RECOM: A code for the simulation of radionuclide resuspension at bubbling water pool surfaces. Proc. Annual Meeting on Nuclear Technology 97, Aachen, Germany, 206-209

Zenz F A and Weil N A 1958 A theoretical empirical approach to the mechanism of particle entrainment from fluidized beds. A.I.Ch.E. J. 4(4): 473 